\title{
Relationship between Inner-Ear Fluid Pressure and Semicircular Canal Afferent Nerve Discharge
}

\author{
A. Yamauchi, ${ }^{1}$ R. D. Rabbitt,${ }^{1,4}$ R. Boyle, ${ }^{2,4}$ and S. M. Highstein ${ }^{3,4}$ \\ ${ }^{1}$ Department of Bioengineering, University of Utah, Salt Lake City, UT 84112, USA \\ ${ }^{2}$ NASA Ames Center for Bioinformatics, Moffett Field, CA 94035, USA \\ ${ }^{3}$ Department of Otolaryngology and Neurobiology, Washington University, St. Louis, MO, 62103, USA \\ ${ }^{4}$ Marine Biological Laboratory, Woods Hole, MA, USA
}

Received: 9 November 2000; Accepted: 10 May 2001; Online publication: 31 August 2001

\begin{abstract}
The present study was designed to determine (1) the transcupular fluid pressure $(\Delta P)$ generated across the semicircular canal cupula in response to sinusoidal head rotation, (2) the translabyrinthine dilational pressure $\left(P_{0}\right)$ generated across the membranous labyrinth in response to an increase in endolymph fluid volume (hydrops), (3) afferent nerve discharge patterns generated by these distinct pressure stimuli and, (4) threshold values of $\Delta P$ and $P_{0}$ required to elicit afferent neural responses. The experimental model was the oyster toadfish, Opsanus tau. Micromechanical indentation of the horizontal canal (HC) duct and utricular vestibule was used to simulate sinusoidal head rotation and fluid volume injection. Single-unit neural spike trains and endolymph pressure within the ampulla, on both sides of the cupula, were recorded simultaneously. $\Delta P$ averaged $0.013 \mathrm{~Pa}$ per $1^{\circ} \%$ s of sinusoidal angular head velocity and $P_{0}$ averaged $0.2 \mathrm{~Pa}$ per $1 \mathrm{~nL}$ of endolymph volume injection. The most responsive afferents had a threshold sensitivity to $\Delta P$ of $10^{-3} \mathrm{~Pa}$ and to $P_{0}$ of $5 \times 10^{-2} \mathrm{~Pa}$ based on a discharge modulation criterion of 1 impulse/s per cycle for 2 $\mathrm{Hz}$ pressure stimuli. Neural sensitivity to $\Delta P$ was expected on the basis of transverse cupular and hair bundle deflections. Analysis of mechanics of the end organ,
\end{abstract}

Abbreviations: $1 \mathrm{~Pa}=1 \mathrm{~N} / \mathrm{m}^{2}=10 \mathrm{dyn} / \mathrm{cm}^{2} \approx 102 \mu \mathrm{m} \mathrm{H}_{2} \mathrm{O} \approx$ $0.0075 \mathrm{~mm} \mathrm{Hg} . \mathrm{nL}=$ nanoliter

Correspondence to: Richard D. Rabbitt, Ph.D. - Department of Bioengineering, $2480 \mathrm{MEB} \cdot$ University of Utah -50 South Central Campus Drive - Salt Lake City, UT 84112. Telephone: (801) 5816968; fax: (801) 585-5361; email: r.rabbitt@utah.edu neuronal projections into the crista, and individual neural firing patterns indicates that $P_{0}$ sensitivity resulted from pressure-induced distension of the ampulla that led to a nonuniform cupular deformation pattern and hair bundle deflections. This explanation is consistent with predictions of a finite element model of the end organ. Results have implications regarding the role of $\Delta P$ in angular motion transduction and the role of $P_{0}$ under transient hydropic conditions.

Keywords: mechanoelectric transduction, endolymphatic hydrops, vestibular mechanics

\section{INTRODUCTION}

Auditory and vestibular end organs exhibit morphological specializations which tune their sensitivities to particular forms of mechanical vibration and/or gravito-inertial acceleration. In the case of vertebrate semicircular canals and the mammalian cochleas, the influence of fluid-compartment morphology on mechanics is critical to the sensing process. An important mechanical variable is fluid pressure, which provides the primary force driving motion of the cochlear partition and the semicircular canal cupula. Although dynamic fluid pressure changes are an integral part of inner-ear physiology, direct experimental measurement of fluid pressure modulations caused by physiological stimuli has proven difficult.

Inner-ear fluid pressure data reported to date fall primarily within two extreme stimulus regimes: (1) acoustic frequencies appropriate for study of cochlear physiology (Lynch et al. 1982; Olson 1998; Puria et 
al. 1997; Dancer and Franke 1980) or (2) quasistatic endolymph pressure increases observed during surgically induced endolymphatic hydrops or fluid injection into inner-ear fluid compartments (Bohmer 1993; Horner 1993; Salt and DeMott 1997; Wit et al. 2000). The goals of the present study were to measure physiological pressure modulations within the ampulla of the semicircular canal and to correlate components of the pressure with afferent nerve discharge patterns. Achievement of these goals required measurement of endolymph pressure modulation of $\left(10^{-3} \mathrm{~Pa}\right.$ in situ over a frequency range of $0.4-20 \mathrm{~Hz}$-sensitivity beyond the capability of traditional servo-driven micropipette systems or fiber-based microphones (Andrews et al. 1991; Bohmer 1993; Olson 1998). Therefore, it was necessary to develop and apply new pressure measurement technology (see Methods).

Two pressure probes were used simultaneously to measure endolymph pressure on the horizontal canal $(\mathrm{HC})$ lumen side of the cupula $\left(P_{\mathrm{HC}}\right)$ and on the utricular side of the cupula $\left(P_{\mathrm{U}}\right)$. Endolymph pressure recordings were made relative to perilymph, which was exposed to atmospheric pressure, and used to determine the transcupular pressure $\left(\Delta P=P_{\mathrm{HC}}-P_{\mathrm{U}}\right)$ and the dilational pressure $\left[P_{0}=\left(P_{\mathrm{HC}}+P_{\mathrm{U}}\right) / 2\right]$. The transcupular pressure $\Delta P$ is the primary macromechanical force driving transverse displacement of the cupula and thus is the primary force leading to hair bundle deflection. As a result, $\Delta P$ has more physiological relevance than $P_{0}$ at least under normal conditions. Measurement of $\Delta P$ and the associated neural threshold defines the first objective of the study.

There are conditions, however, where $P_{0}$ may also be important. Indirect evidence suggests that some semicircular canal afferent nerves may respond to modulation of $P_{0}$ even in the complete absence of $\Delta P$ (Rabbitt et al. 1994, 1995). The origin of this apparent dilational pressure sensitivity was not previously proven, but it was hypothesized that changes in $P_{0}$ might cause distension of the membranous ampulla wall, thus leading to deformation of the cupula and the associated neural response. Clinical studies of the pathological condition of endolymphatic hydrops have also raised the possibility that cupular deformation might be induced by changes in endolymphatic pressure (Andrews and Honrubia 1996; Tonndorf 1983). There remains an ongoing debate as to whether or not any significant fluid pressure or mechanical activation of hair cells accompanies Ménière's diseaserelated endolymphatic hydrops (Honrubia 1999; Salt 1999; Wit et al. 2000). Direct measurement of the neural threshold to $P_{0}$ therefore defines the second objective of the study. The physiological origin of $P_{0}$ sensitivity is elucidated herein through analysis of individual afferent responses and the cupular deformation field.

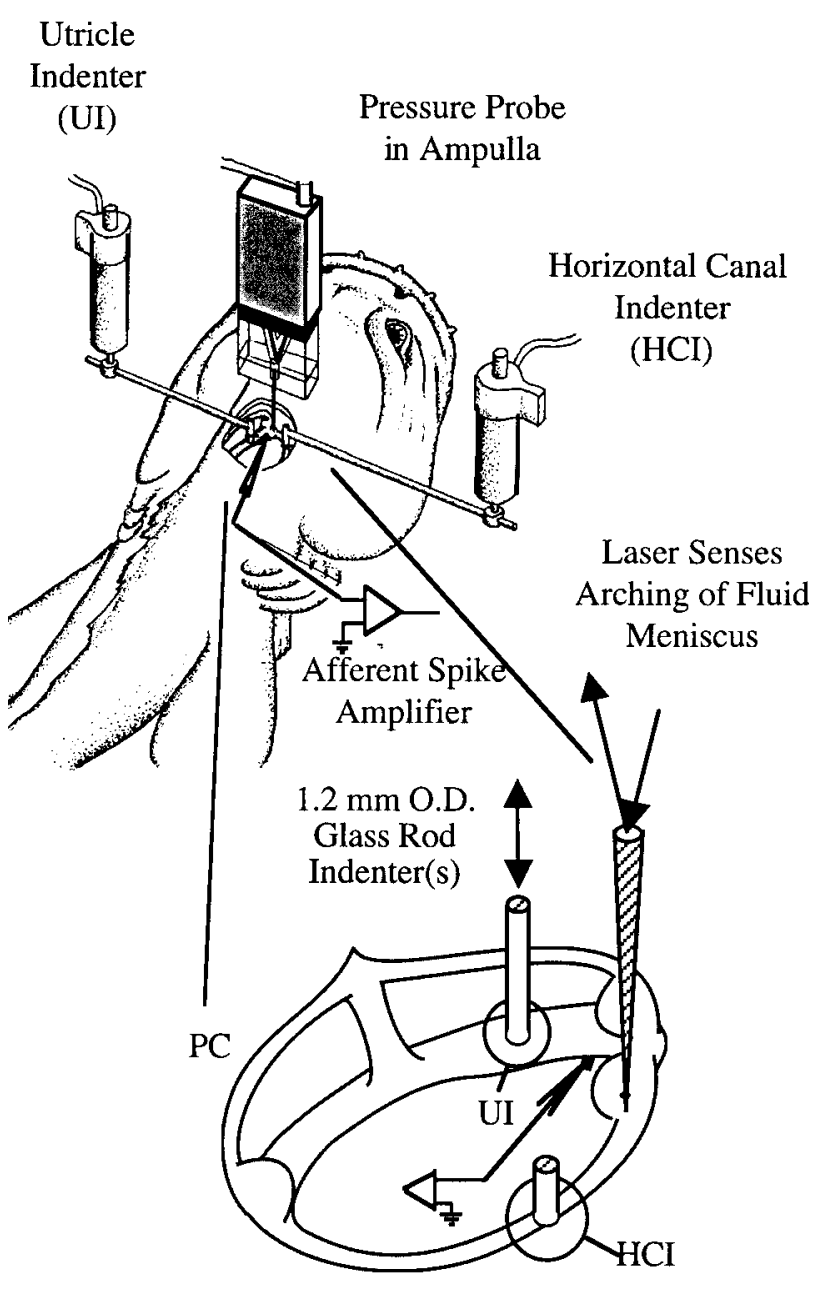

FIG. 1. Fish preparation. A toadfish was secured in a plastic box with the gills and two-thirds of the body immersed in seawater. A small craniotomy exposed the horizontal canal and nerve. Laserpipette pressure probes were inserted into small fistulas in the horizontal ampulla on the canal lumen and utricular sides of the cupula. Mechanical stimulators were placed on the horizontal canal duct $(\mathrm{HCl})$ and the utricle (UI). Glass microelectrodes were used for horizontal canal nerve afferent recording.

\section{METHODS}

\section{Fish preparation}

(Fig. 1) Twelve adult toadfish (Opsanus tau) provided useful pressure and afferent data, and an additional six provided useful afferent data. In preparation for surgery, the fish was anesthetized with MS222 (5 mg/ $\mathrm{L}$ in seawater) and partially immobilized by an intramuscular injection of pancuronium bromide $(0.05$ $\mathrm{mg} / \mathrm{kg}$ ). The fish was then secured in a plastic tank, with two-thirds of its body immersed in bubbled seawater. The remainder of the body was covered with moist tissues. A small craniotomy was made lateral to the dorsal course of the anterior canal and rostral to the common crus to expose the horizontal and anterior 
canal ampullae, a portion of the utricle, and $8 \mathrm{~mm}$ of the HC ampulla. During surgery, perilymph within the upper region of the cavity was replaced with fluorocarbon (FC-75, 3M Corp. St. Paul, MN, USA). The entire HC nerve remained bathed in normal perilymph; and the partial fluid change did not alter afferent responses. To allow measurement of endolymphatic pressure, two small holes were made in the horizontal canal ampulla wall, 300-500 $\mu \mathrm{m}$ from each side of the cupula, using an electrosurgical generator (SSE4, Valleylab, Boulder, CO, USA). A $76 \mu \mathrm{m}$ diameter tungsten wire, serving as the cutting electrode, was insulated with a glass pipette and lowered onto the ampullary surface with a micromanipulator. The endolymphatic fistula was made by brief delivery of a 4-8 $\mathrm{W}$ cutting waveform using manual footswitch control. This generated a 25-75 $\mu \mathrm{m}$ diameter hole in the membranous ampullary wall, allowing access to the endolymph. Neural responses were recorded before and after generation of the fistula in control animals to ensure normal function.

\section{Stimulus}

Ewald (1892) introduced mechanical indentation of the slender limb of the membranous canal as a stimulus to produce deflection of the cupula and semicircular canal neural responses in the absence of head rotation (Camis 1930). It was later shown by Hillman and McLaren (1979) that mechanical indentation displaces endolymph away from the stimulation site and can be used to induce maintained cupular deflections in plug-canal preparations. The mechanical stimulus was later refined by Dickman et al. (1988) for application in patent canals and shown to elicit robust responses of single semicircular canal afferent neurons. Dickman et al. (1989a, b) were able to show that sinusoidal indentation of the canal duct could be used to mimic physiological sinusoidal angular head velocity and produce nearly equivalent afferent discharge patterns. The origin of this correspondence was subsequently quantified in terms of the mechanics of the deformable membranous labyrinth and endolymph flow (Rabbitt et al. 1995, 1999). In short, local indentation of the duct is equivalent to injecting a small bolus of fluid into the canal at the site of the indentation. Some of the fluid is displaced toward the ampulla which ultimately leads to deflection of the cupula and afferent discharge modulation. In the oyster toadfish, $1 \mu \mathrm{m}$ of sinusoidal mechanical indentation of the horizontal canal limb has been shown to generate afferent responses nearly equivalent to those generated by $4^{\circ} \%$ $\mathrm{s}$ of sinusoidal angular head velocity (with frequency limitations; Rabbitt et al. 1995). Equivalence of afferent discharge patterns for the two stimuli implies nearly equivalent cupular deflections and associated transcupular pressure gradients $(\Delta P)$. There are, however, slight differences in afferent responses for indentation vs. rotation, even at low stimulus frequencies (Dickman and Corriea 1989b; Rabbitt et al. 1994, 1995, 1999). These relatively small differences have been hypothesized to arise from the influence of dilational pressure $P_{0}$ that is present during mechanical indentation of the labyrinth but nearly absent during physiological head rotation (Rabbitt et al. 1994, 1999; Hess et al. 2000).

Mechanical indentation of the canal duct was used in the present study to generate $\Delta P$ and $P_{0}$ following the approach described previously by Rabbitt et al. (1995). Briefly, $1.2 \mathrm{~mm}$ diameter glass rods were used to indent the horizontal canal duct and/or the utricle. Each rod was attached to a piezoelectric microactuator (PZL 060-11, Burleigh, Victor, NY, USA), and its displacement was monitored using a linear variable differential transformer (DEC-050, Schaevitz, Slough Berks, UK) mounted in-line with the actuator. For endolymphatic pressure measurements, one stimulator was used to indent the long-and-slender portion of the horizontal canal. The mechanical stimulus consisted of a 2-15 $\mu \mathrm{m}$ zero-to-peak sinusoidal indentation of the horizontal canal, at frequencies between 0.4 and $20 \mathrm{~Hz}$. The stimulus was also applied while the pressure sensor was being positioned in order to monitor the sensor function (described below). In some experiments, one indenter each was placed on the horizontal canal duct and the utricle for combined stimuli. On the horizontal canal duct, the indenter was placed 6 $\mathrm{mm}$ from the cupula along the curved centerline of the canal and preloaded to a static indentation of 12 $\mu \mathrm{m}$. On the utricle, the indenter was placed $3 \mathrm{~mm}$ from the cupula and preloaded to a static indentation of $25 \mu \mathrm{m}$ (Rabbitt et al. 1995). Indentation stimuli were then applied by sinusoidal command signals (AFG 5102, Tektronix, Beaverton, OR, USA) delivered to the piezoelectric actuators via a high-voltage amplifier (207 A, TrigTek, Anaheim, CA, USA). In some experiments, the amplitudes of the HC and utricular stimuli were balanced against each other, as described by Rabbitt et al. (1994), to achieve equal but oppositephase afferent responses when applied separately. The balanced $\mathrm{HC}$ and utricular stimuli were applied together at $1-2 \mathrm{~Hz}$ to minimize $\Delta P$ and generate dilational " $P_{0}$ stimulation."

\section{Neural recording}

Horizontal canal neural recordings used glass microelectrodes $(60 \mathrm{M} \Omega)$ filled with $3 \mathrm{M} \mathrm{NaCl}$ or $\mathrm{LiCl}_{2}$ following the approach described previously (Boyle and Highstein 1990). Electrodes were positioned using a micromanipulator at a location $1 \mathrm{~mm}$ from the horizontal canal ampulla, where a portion of the HC nerve 


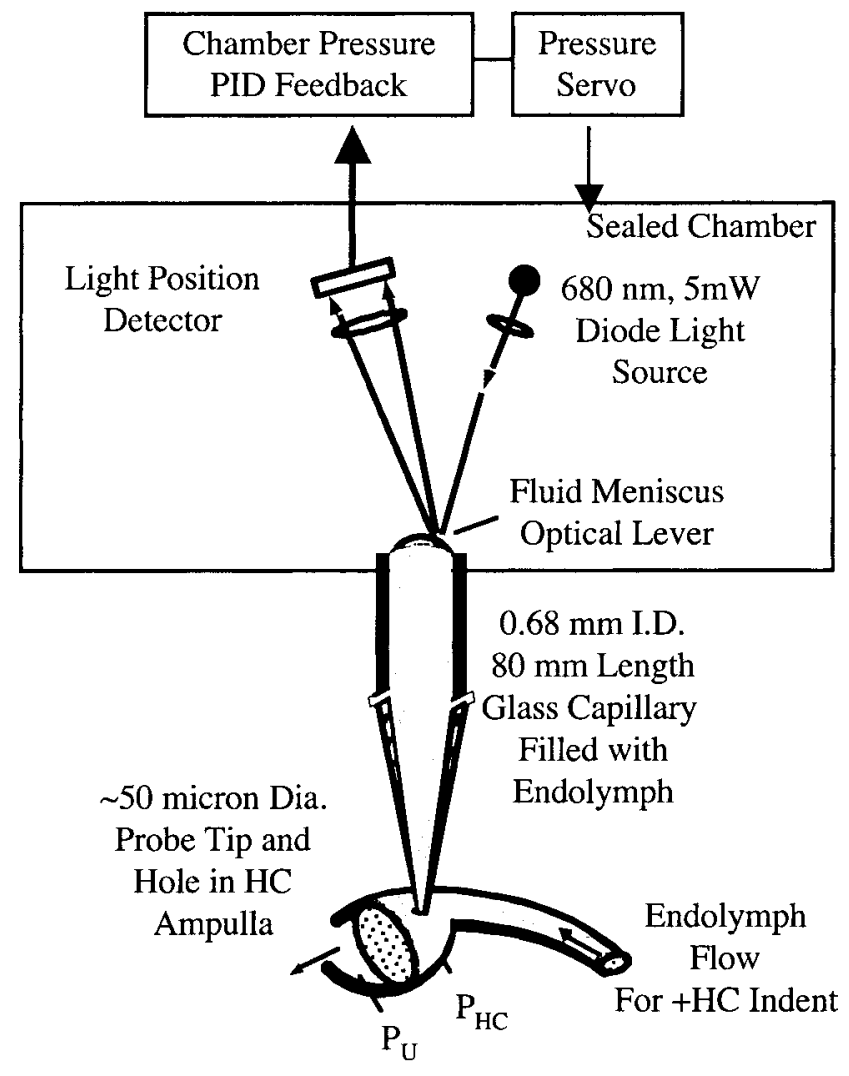

FIG. 2. Laser-pipette pressure sensor. The pressure probes consisted of a endolymph-filled micropipette coupled to a laser displacement sensor. Pressure caused slight bowing of the meniscus that was sensed by optical lever deflection of the laser beam. A proportional-integralderivative (PID) pressure feedback loop was employed to adjust the pressure acting on the meniscus and compensate for external atmospheric pressure fluctuations.

was accessible. Extracellular potentials were measured using standard bridge amplification and external spike discrimination.

\section{Pressure sensor}

Endolymphatic pressure modulations in the ampulla, resulting from application of the mechanical stimuli, were measured using a custom-built pressure measurement system (Fig. 2). The probe consisted of a glass micropipette that was pulled and cut to a tip diameter (25-75 $\mu \mathrm{m}$ to closely match the endolymphatic fistula in each fish. The glass probe was filled with artificial endolymph (AE), as described below, and inserted into the microcauterized hole under manual micromanipulator guidance. A laser displacement sensor (LAS2010, Nippon Automation, Hamamatsu, Shizuoka, Japan) was used to deliver focused light to the fluid meniscus at the top of the micropipette. The curved surface of the meniscus was used as an optical lever, such that small changes in pressure at the tip of the pipette caused large deflections in the angle of the reflected laser light beam. The change in the angle of the reflected light was monitored using a light-position-sensitive detector. The laser source/detector unit was held in a fixed position relative to the pipette using a clear acrylic chamber. The chamber was attached to the laser case using silicone sealant (Elmer's E-920, Elmer's Products, Inc., Columbus, $\mathrm{OH}$ ). The pipette shank was secured to the bottom of the chamber using a patch-clamp-style holder. This created a sealed air chamber between the pipette shank and the laser case that exerted a "reference pressure" on the free surface of the fluid meniscus (see Fig. 2). The static reference pressure was adjusted in order to position the fluid meniscus to balance the weight of the column of endolymph within the pipette, the capillary force of the fluid moving up the pipette, and the static pressure at the probe tip. Pressure perturbations were recorded relative to this statically balanced configuration. Procedures for filtering and balancing the probe are described below.

Since the fluid meniscus at the top of the pipette was in a sealed chamber, the pressure measurement system was single ended so that recordings in endolymph were sensitive to perturbations in atmospheric pressure which influenced the hydrostatic pressure of the perilymph and endolymph. Random changes in the ambient pressure were larger than the endolymph pressure modulations of interest in our study and, without attention, would have saturated the sensitive probe. Hence, it was necessary to devise a means to reduce the influence of ambient pressure fluctuations. To reduce the unwanted signals, the pressure within the sealed acrylic chamber above the micropipette was regulated using a PID (proportional-integral-derivative) feedback loop (SuperScopeII, GW Instruments, Cambridge, MA). The pressure changes were accomplished by control of a reversible DC motor which advanced and retracted a fine micrometer screw against a rubber diaphragm connected to the acrylic chamber by PVC tubing. This feedback was employed to avoid ambient pressure-induced sensor saturation. A high-pass approach was used such that the feedback system canceled only the low-frequency pressure fluctuations $(<0.4 \mathrm{~Hz})$. The dynamic response of the sensor was calibrated in situ, as described below, with the feedback in operation. This provided a useful recording range of $0.4-20 \mathrm{~Hz}$. Measurements $>20 \mathrm{~Hz}$ were limited as a result of the gain roll-off caused by the fluid mass and viscosity in the probe micropipette. Fourier methods were used to lock in the stimulus frequency and further reduce any uncorrelated pressure fluctuations (see below).

Immediately before the micropipette tip was placed into the ampullary fistula, the FC-75 fluid level was lowered below the ampullary fistula and a thin layer of $\mathrm{AE}$ was injected into the cavity to cover the hole. 


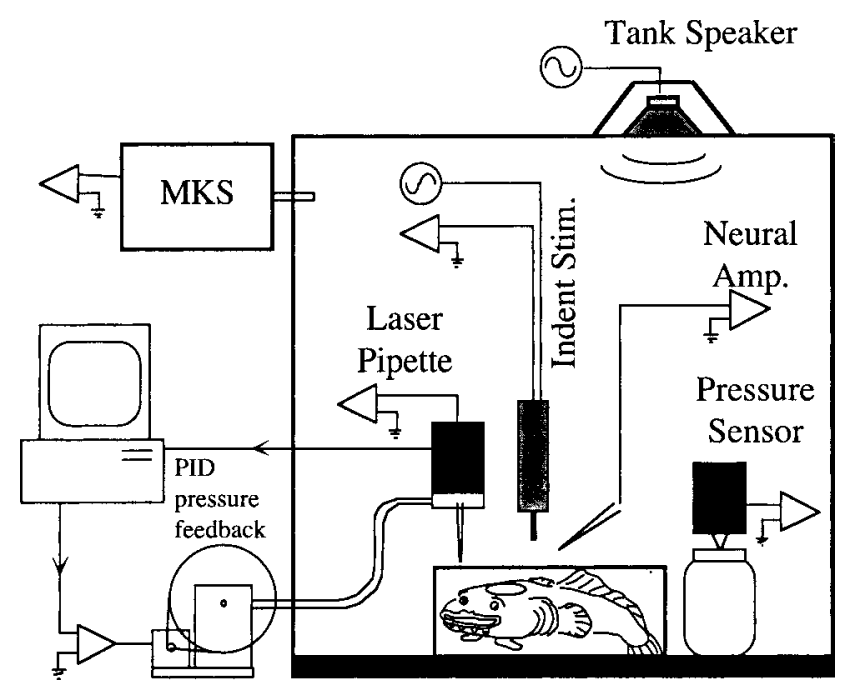

FIG. 3. Calibration tank and instrumentation. The entire apparatus was placed in a large plastic tank for calibration of the laser-pipette pressure probes. A loudspeaker mounted into the top of the tank was used to modulate the pressure within the tank and throughout the animal's body. A pressure feedback system was used to control the reference pressure acting on the pipette meniscus surface. Recording of tank pressure modulation (MKS and diaphragm units) and laserpipette voltage outputs during tank pressure stimuli (speaker) allowed for calibration of the laser-pipette probes in situ.

The high specific gravity and immiscibility of FC-75 served to isolate this thin layer of $\mathrm{AE}$ from the perilymph. The micropipette was then placed into the $\mathrm{AE}$ layer to allow the feedback system to equilibrate the probe-tip pressure with the $\mathrm{AE}$ pressure just prior to lowering the probe into the ampulla. This prevented fluid movement into or out of the pipette while lowering it into the fistula. Proper placement of the micropipette was confirmed by microscopic observation and by the responsiveness of the pressure probe to $\mathrm{HC}$ mechanical indentation. After inserting the probe, the $\mathrm{AE}$ was quickly removed and the instrumented ampulla was immediately covered with FC-75. The FC75 is not miscible with perilymph or endolymph, thus, it formed a surface tension seal around the pipette at the point of insertion into the ampulla. The effectiveness of the surface tension seal was confirmed in control experiments by comparing pressure modulations before and after sealing the micropipette into the hole using Super Glue.

Each pressure pipette was calibrated in situ. After the pressure probe was positioned in the ampulla, the entire apparatus was sealed inside of a 23 in. $\times 28$ in. $\times 32$ in. Plexiglas box (see Fig. 3). The pressure within the sealed tank was modulated using pure sinusoids delivered to a loudspeaker secured in the top wall of the tank. For the low-frequency stimuli used, the tank was excited in the 0th acoustic mode such that the pressure in the tank was spatially homogeneous and modulated in temporal synchrony. This stimulus placed the fish in a modulating hydrostatic pressure field and hence generated internal endolymphatic pressure fluctuations identical to the internal tank pressure fluctuations (confirmed by multiple probe measurements). The magnitude of the pressure within the box was measured using a differential pressure transducer (MKS 698, Type 270 Signal Conditioner, Andover, MA, USA) pressure sensor in Fig. 3). The MKS pressure-sensing system exhibited an unacceptable phase shift above $0.8 \mathrm{~Hz}$. Therefore, we also monitored the pressure inside the box by measuring the displacement of a thin latex membrane stretched over a large air container (Fig. 3), membrane-type tank pressure sensor). Membrane displacement was measured with a laser displacement sensor. The phase of the membrane corresponded well with the MKS unit below $0.8 \mathrm{~Hz}$. By comparing the phase of speaker displacement to the membrane pressure sensor it was determined that the membrane faithfully followed the phase of the pressure in the tank over the bandwidth employed in the present experiments. This allowed us to calibrate the membrane-type sensor using the lowfrequency MKS output. After calibration, the membrane-type sensor was used to determine the magnitude and phase of the tank pressure. The complexnumber representation of the measured tank pressure (in $\mathrm{Pa}$ ) was divided by the analog output of the laserpipette pressure probe (in $\mathrm{V}$ ) to determine the calibration transfer function $\left[T_{c}(\omega)\right.$ in $\left.\mathrm{Pa} / \mathrm{V}\right]$ for the pressure sensor in situ. This was done in the frequency domain by signal averaging 10-100 cycles of the sinusoidal response at 20 discrete frequencies between 0.2 and $20 \mathrm{~Hz}$. Calibration was carried out independently for each probe to account for changes in pipette-probetip geometry and probe insertion.

\section{Data acquisition}

Digital data acquisition (Cambridge Electronic Design 1401 Plus, Spike2, Apple Macintosh interface) was used to record endolymph pressure (laser-pipette probes), displacement of the indenters (LVDTs), spike times of afferent discharge, and tank pressure (MKS and laser diaphragm sensor). The analog signals were externally amplified to span the 12 bit range of the A/D converter, filtered at $120 \mathrm{~Hz}$, and sampled at 250 Hz. Spike times were externally discriminated (74-60-4, FHC, Brunswick, ME, USA). Spike times and stimulus trigger signals, used to drive the piezoelectric actuators and the tank speaker, were recorded with a temporal resolution of $0.08 \mathrm{~ms}$.

\section{Data analysis}

First- and second-harmonic gain and phase of afferent responses were determined using constrained Fourier 
methods as described previously (Highstein et al. 1996; Rabbitt et al. 1996). Briefly, the stimulus trigger for indentation was used to produce $100 \mathrm{bin} /$ cycle phase histograms of the neural response. The histograms were normalized by the number of cycles being evaluated such that results were not sensitive to the number of cycles employed. Three-point box smoothing was applied for data display. The raw histogram was fitted to a sine wave at the stimulus frequency and twice the stimulus frequency. Empty bins were ignored in the fitting procedure and the DC firing rate was restricted to be $>0$. This was used to determine the first- and second-harmonic afferent responses. Complex-number representations of neural firing rates were divided by the corresponding stimulus to determine the afferent gain (impulses/s per $\mu \mathrm{m}$ ) and phase (deg re: peak indent). Some semicircular canal afferent responses exhibit cutoff for inhibitory stimuli and/or saturating nonlinearity at high excitatory stimulus levels (Fernández and Goldberg 1971). Present mechanical indentation stimuli were maintained below $6 \mu \mathrm{m} \mathrm{rms-a}$ range where afferents in the toadfish respond nearly linearly for the excitatory phase of the stimulus (Boyle and Highstein 1990; Rabbitt et al. 1995, 1996).

Discrete Fourier analysis was employed for all ana$\log$ signals. Waveform averaging was applied over 10 50 cycles of the sinusoidal stimulus to determine the complex-number representation of laser-pipette voltage (V) for each stimulus frequency. This laser-pipette voltage was multiplied by the corresponding laserpipette calibration transfer function $\left[T_{c}(\omega), \mathrm{Pa} / \mathrm{V}\right]$ on a frequency-specific basis to determine the complexnumber representation of the pressure $(\mathrm{Pa})$. The complex-number representation of the pressure modulation was divided by the stimulus to arrive at the gain $(\mathrm{Pa} / \mu \mathrm{m})$ and phase (deg re: peak indent) of endolymphatic pressure modulation re: indent. Pressure amplitude and phase on the $\mathrm{HC}$ and utricular sides of the cupula $\left(P_{\mathrm{HC}}\right.$ and $\left.P_{\mathrm{U}}\right)$ were found to be nearly linear over the range of indentation amplitudes $(0.5-15 \mu \mathrm{m})$ and frequencies tested, allowing data to be reported in linear Bode form of gain and phase as a function of frequency.

Transcupular and dilational pressure data exhibited nearly constant phase and relatively weak increases in gain with frequency over the bandwidth tested. This weak frequency dependence was fit using a simple transfer function of the form $T(s)=c s^{m}$, where $s$ is the Laplace variable, $c$ is a constant, and $m$ is the gain exponent (i.e., fractional Laplace power). Curve fitting was done in the complex frequency domain with equal weighting to the real and to the imaginary components of the transfer function (Igor Pro, Wave Metrics, Oswego, OR). The standard deviation of the gain exponent $m$ and amplitude $c$ were estimated from the

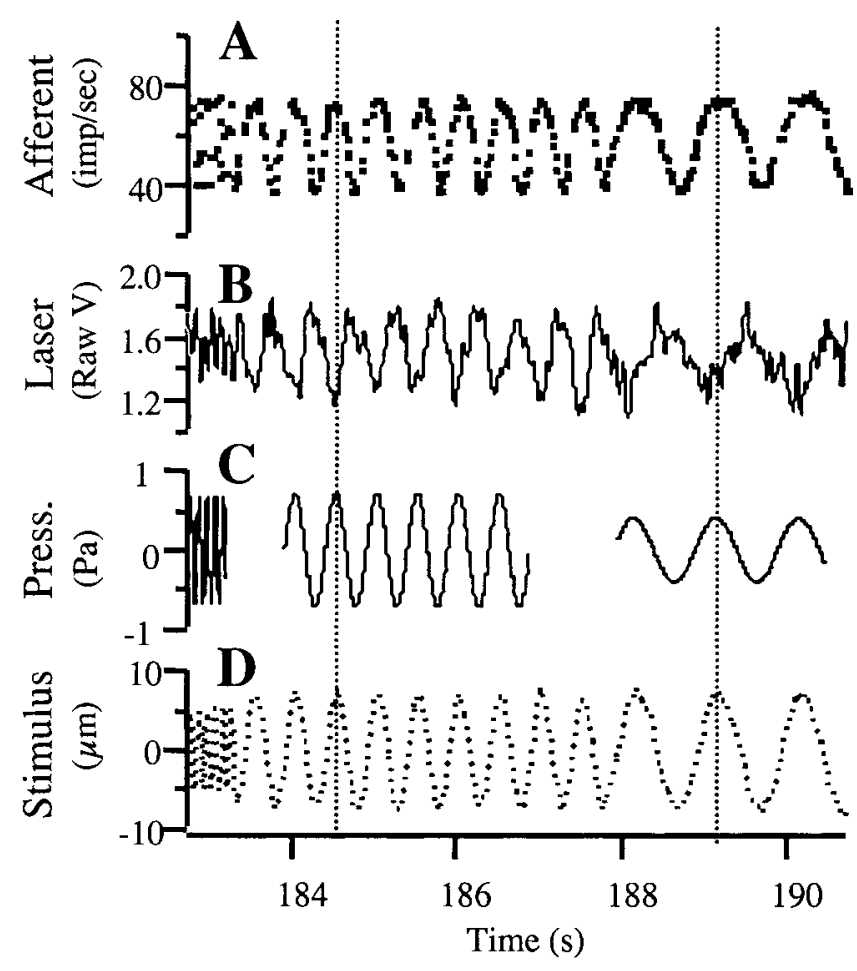

FIG. 4. Raw pressure and afferent data. Pressure measurements and neural recordings were made simultaneously. A Instantaneous spike frequency (impulses/s), B raw laser-pipette pressure probe voltage output, $\mathbf{C}$ first-harmonic pressure after application of the calibration transfer function $(\mathrm{Pa})$, $\mathbf{D}$ stimulus, mechanical indentation of the horizontal canal duct $(\mu \mathrm{m})$.

covariance matrix computed at the final iteration of the nonlinear least squares procedure. The associated gain $G(\mathrm{~Pa} / \mu \mathrm{m})$ and phase $\theta(\mathrm{rad})$ for this transfer function are $\log [G(\omega)]=m \log (\omega)+b$ and $\theta=m \pi /$ 2 , respectively, where $\omega$ is the frequency $(\mathrm{rad} / \mathrm{s})$ and $b$ is a constant.

\section{RESULTS}

\section{Simultaneous pressure and afferent recordings}

The laser-pipette sensors were used to measure modulation of endolymph pressure within the ampulla induced by sinusoidal mechanical indentation of the HC limb and/or utricular vestibule. In many cases afferent modulations were recorded at the same time to be certain that afferent responses were within the normal range, even with the pressure probes in place. Figure 4 shows an example of simultaneous neural recordings and pressure measurements within the $\mathrm{HC}$ ampulla during mechanical stimulation (A, afferent discharge; B, raw laser voltage; C, first-harmonic pressure; $\mathrm{D}$, mechanical indentation). The vertical dashed 
lines illustrate that the pressure $(\mathrm{C})$ on the $\mathrm{HC}$ lumen side of the cupula increases with positive indentation (D) of the canal duct-a stimulus resulting in deflection of the HC cupula toward the utricle and an excitatory increase in afferent discharge (A). The afferent shown had a peak response nearly in phase with peak mechanical indentation. Other afferent types showed phase advances up to $90^{\circ}$ re: peak indentation, consistent with previous studies in this species (Boyle and Highstein 1990; Rabbitt et al. 1995).

Afferent responses to HC indentation were also recorded after making the small fistula in the membranous duct but prior to inserting the pressure probe. Afferents continued to modulate within the normal range, indicating that the fistula did not relieve the differential pressure $(\Delta P)$ responsible for transverse cupular deflection. The same insensitivity to making the fistula was noted previously while recording haircell receptor potentials in this preparation (Highstein et al. 1996). These data imply that the intermolecular surface tension between the endolymph inside the ampulla and the FC-75 outside the ampulla was sufficient to effectively seal the small fistula. In developing the protocol, experiments were conducted to verify that endolymph volume was not affected by the fistula either at rest or during mechanical canal indentation. To test the FC-75 seal, a drop of Super Glue was placed around the micropipette at its insertion through the fistula and pressure responses were compared before and after application of the glue. The glue did not change the pressure data, thus indicating that the seal was adequate without the use of glue. Therefore, the reported results were obtained using a tight-fitting micropipette into the fistula and FC-75 covering the ampulla.

Afferent nerve firing rate was also monitored during changes in the ambient tank pressure with the perilymphatic space opened to air. Afferent nerves did not respond to these global tank pressure modulations, consequently indicating that this type of hyperperilymphatic pressure modulation does not induce deformation of the cupula or deflection of hair bundles. Canal afferent nerves did not change their background discharge, even in the presence of tank pressure increases up to $3.4 \times 10^{4} \mathrm{~Pa}(0.3 \mathrm{~atm})$ - a pressure roughly $10^{7}$ times higher than the threshold of the neural sensitivity to $\Delta P$ reported herein. Insensitivity to ambient tank pressure fluctuations speaks to the design of the vestibular labyrinth. Complete suspension of the membranous labyrinth in perilymph causes any perturbation in perilymph pressure to be transmitted uniformly to endolymph, thus minimizing mechanical deformation of the ampulla and cupulae and avoiding a neural response.

\section{Differential and dilational pressure}

As described in the Methods section, local indentation of the canal duct is equivalent to injection of a small bolus of endolymph at the indentation site. For the current experimental setup, the equivalent volume injection was $0.6 \mathrm{~nL} / \mu \mathrm{m}$ (nanoliters of fluid injection per micron of indentation; Rabbitt et al. 1995). Therefore, local indentation of the duct caused global distension of other regions of the membranous labyrinth and an increase in the dilational pressure $P_{0}$. In addition, some of the flow caused by indentation of the duct moved toward the ampulla and caused a differential pressure $\Delta P$ across the cupula. This differential pressure is known to mimic that present during sinusoidal head rotation (Dickman and Corriea 1989a, b; Rabbitt et al. 1995). Hence, mechanical indentation generated both $\Delta P$ and $P_{0}$ at the same time. This was clearly observed in the pressure data.

Figure 5 shows pressure data for two probes recorded simultaneously in a single animal. Pressure on the HC lumen side of the cupula ( $P_{\mathrm{HC}}$, squares) and on the utricular side ( $P_{\mathrm{U}}$ crosses) is shown. There was no significant nonlinearity in $P_{\mathrm{HC}}$ or $P_{\mathrm{U}}$ as the amplitude of the HC indentation stimulus was adjusted from 3 to $18 \mu \mathrm{m}$ (zero to peak), therefore, data are presented in linear Bode form of gain $(\mathrm{A}, \mathrm{Pa} / \mu \mathrm{m})$ and phase $\left(\mathrm{B},{ }^{\circ}\right.$ re: peak indent). Consider the data points at $2 \mathrm{~Hz}$ as examples. Note that the magnitude of $P_{\mathrm{HC}}$ exceeds the magnitude of $P_{\mathrm{U}}$; hence, there was a positive transcupular pressure gradient during positive indentation $\left(\Delta P=P_{\mathrm{HC}}-P_{\mathrm{U}}>0\right)$. This pressure gradient serves to drive the cupula toward the utricle. Also note that the phase of $P_{\mathrm{HC}}$ was slightly greater than the phase of $P_{\mathrm{U}}$; hence, the transcupular pressure had a phase leading that of the mechanical stimulus. The transcupular pressure $\Delta P=P_{\mathrm{HC}}-P_{\mathrm{U}}$ and dilational pressure $P_{0}=\left(P_{\mathrm{HC}}+P_{\mathrm{U}}\right) / 2$ are shown on the right side of Figure 5 as functions of frequency (C gain, $\mathrm{D}$ phase). The $\Delta P$ gain for this fish increased by a factor of 3 over the stimulus frequency bandwidth. Fitting the data with a simple fractional order transfer function (see Methods and Table 1) provided a gain exponent of $m_{\triangle P}=0.33( \pm 0.032 \mathrm{SD})$ and an amplitude of $c_{\Delta P}=0.015( \pm 0.0018 \mathrm{SD})$ for this animal. Gain and phase of the $\Delta P$ transfer function are shown as solid (A) and dashed lines (B), respectively. The exponent $m_{\Delta P}=0.33$ is the slope of the gain on a log-log scale $\left[m_{\Delta P}=\Delta \log\right.$ (gain) $\left./ \Delta \log (\omega)\right]$ and corresponds quantitatively to a constant phase $\theta_{\Delta P}=m_{\Delta P} \pi / 2\left(29^{\circ}\right)$. The fitting procedure optimized the real and imaginary components of the transfer function, and, therefore, optimized parameters are based on both gain and phase. The average phase over the frequency bandwidth tested was $\bar{\theta}=28^{\circ}$, a value that corresponds well with the optimized value of $\theta_{\Delta P}=29^{\circ}$. Linear 
TABLE 1

Transfer functions relating mechanical indentation to $\Delta P$ and $P_{0}$ as functions of the Laplace variable $s$

\begin{tabular}{|c|c|c|c|c|}
\hline \multirow[b]{2}{*}{ Animal } & \multicolumn{2}{|c|}{$\begin{array}{c}\text { Transcupular Pressure } \Delta P \\
T_{\Delta P}=c_{\Delta P} S^{m_{\Delta P}}(\mathrm{~Pa} / \mu \mathrm{m})\end{array}$} & \multicolumn{2}{|c|}{$\begin{array}{l}\text { Dilational Pressure } P_{0} \\
T_{P_{0}}=C_{P_{0}} S^{m} P_{0}(\mathrm{~Pa} / \mu \mathrm{m})\end{array}$} \\
\hline & $m_{\Delta P}$ & $c_{\Delta P}$ & $m_{P_{0}}$ & $C_{P_{0}}$ \\
\hline A & $0.45 \pm 0.15$ & $0.0037 \pm 0.0019$ & $0.15 \pm 0.062$ & $0.013 \pm 0.0028$ \\
\hline B & $0.33 \pm 0.032$ & $0.015 \pm 0.0018$ & $0.0061 \pm 0.021$ & $0.070 \pm 0.0052$ \\
\hline C & $0.45 \pm 0.043$ & $0.013 \pm 0.0022$ & $0.14 \pm 0.031$ & $0.13 \pm 0.016$ \\
\hline $\mathrm{D}$ & $0.23 \pm 0.069$ & $0.095 \pm 0.024$ & $0.14 \pm 0.021$ & $0.35 \pm 0.027$ \\
\hline$E$ & $0.15 \pm 0.023$ & $0.047 \pm 0.0031$ & $0.36 \pm 0.024$ & $0.015 \pm 0.0014$ \\
\hline $\mathrm{F}$ & $0.12 \pm 0.015$ & $0.062 \pm 0.0029$ & $0.28 \pm 0.020$ & $0.022 \pm 0.0016$ \\
\hline G & $0.32 \pm 0.19$ & $0.027 \pm 0.018$ & $0.0061 \pm 0.036$ & $0.31 \pm 0.035$ \\
\hline $\mathrm{H}$ & $0.15 \pm 0.058$ & $0.048 \pm 0.012$ & $0.018 \pm 0.023$ & $0.35 \pm 0.029$ \\
\hline I & $0.41 \pm 0.050$ & $0.011 \pm 0.0022$ & $0.044 \pm 0.032$ & $0.24 \pm 0.028$ \\
\hline J & $0.25 \pm 0.032$ & $0.044 \pm 0.0059$ & $0.38 \pm 0.054$ & $0.018 \pm 0.0042$ \\
\hline K & $0.21 \pm 0.030$ & $0.010 \pm 0.0012$ & $0.33 \pm 0.068$ & $0.0071 \pm 0.0018$ \\
\hline $\mathrm{L}$ & $0.45 \pm 0.10$ & $0.0036 \pm 0.0016$ & $0.24 \pm 0.050$ & $0.025 \pm 0.0051$ \\
\hline Avg. & $0.29 \pm 0.067$ & $0.031 \pm 0.0082$ & $0.17 \pm 0.037$ & $0.13 \pm 0.013$ \\
\hline
\end{tabular}

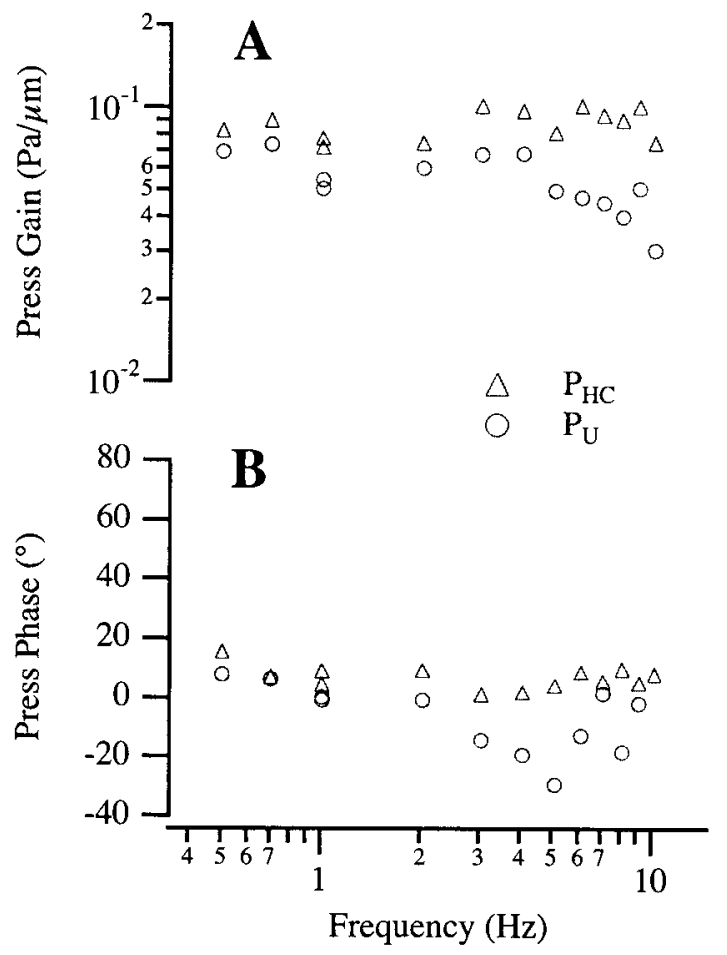

FIG. 5 Pressure Bode plots for a single animal. The pressures recorded simultaneously on the $\mathrm{HC}$ lumen side of the cupula $\left(P_{\mathrm{HC}}\right)$ and on the utricular side of the cupula $\left(P_{\mathrm{U}}\right)$ are shown for a representative fish in response to mechanical indentation of the canal duct $(\mu \mathrm{m})$. The gain $(\mathbf{A}, \mathrm{Pa} / \mu \mathrm{m}$ indent) and phase (B, deg re: peak indent) are shown as functions of indentation frequency. The gain $(\mathbf{C})$ and

regression revealed a strong correlation between $\bar{\theta}$ and $\theta_{\Delta P}$ for the population as a whole $(R=0.87)$, thus supporting use of the simple fractional Laplace transfer function as a reasonable empirical description of the data.

The gain exponent $(m)$ and the phase $(\theta)$ measure

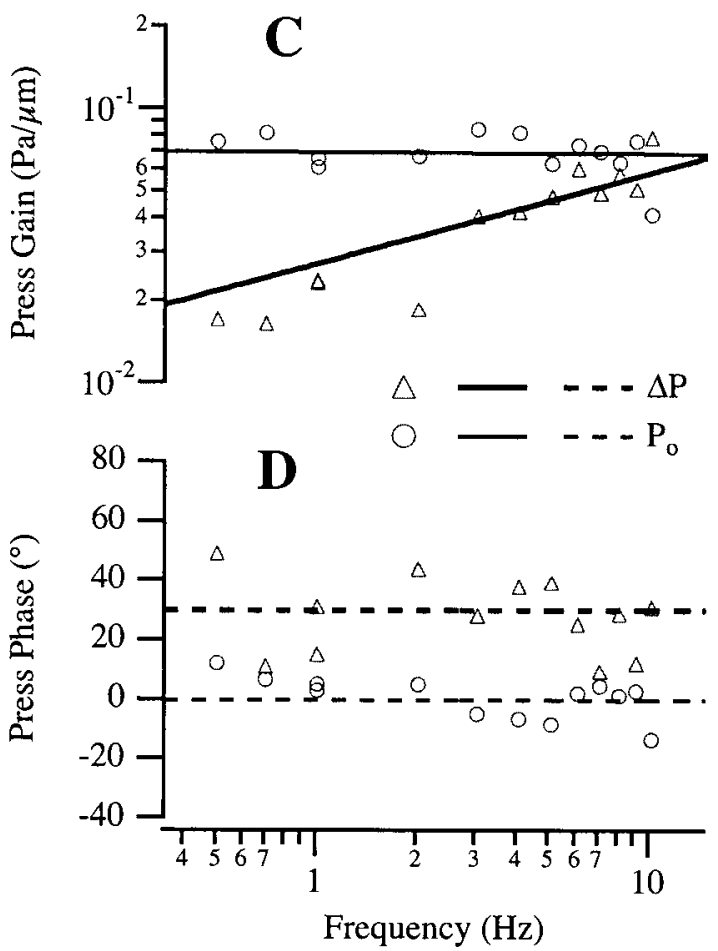

phase (D) of the transcupular pressure $\Delta P$ and the dilational pressure $P_{0}$ are shown in the right panel for the same animal [note: $\Delta P=P_{\mathrm{HC}}$ $-P_{\mathrm{U}}$ and $\left.P_{0}=\left(P_{\mathrm{HC}}+P_{\mathrm{U}}\right) / 2\right]$. Solid (gain) and dashed (phase) lines show results of least-squares linear fits to $\Delta P$ and $P_{0}$ (thin) using a of a simple fractional-power transfer function (animal B, Table 1).

to what extent the pressure was proportional to indentation displacement $\left(m=0, \theta=0^{\circ}\right)$ or indentation velocity $\left.(m=1, \theta=\pi / 2) \mathrm{rad}, 90^{\circ}\right)$. Proportionality to displacement corresponds to a mechanical response dominated by mechanical stiffness, while proportionality to velocity corresponds to a response dominated 

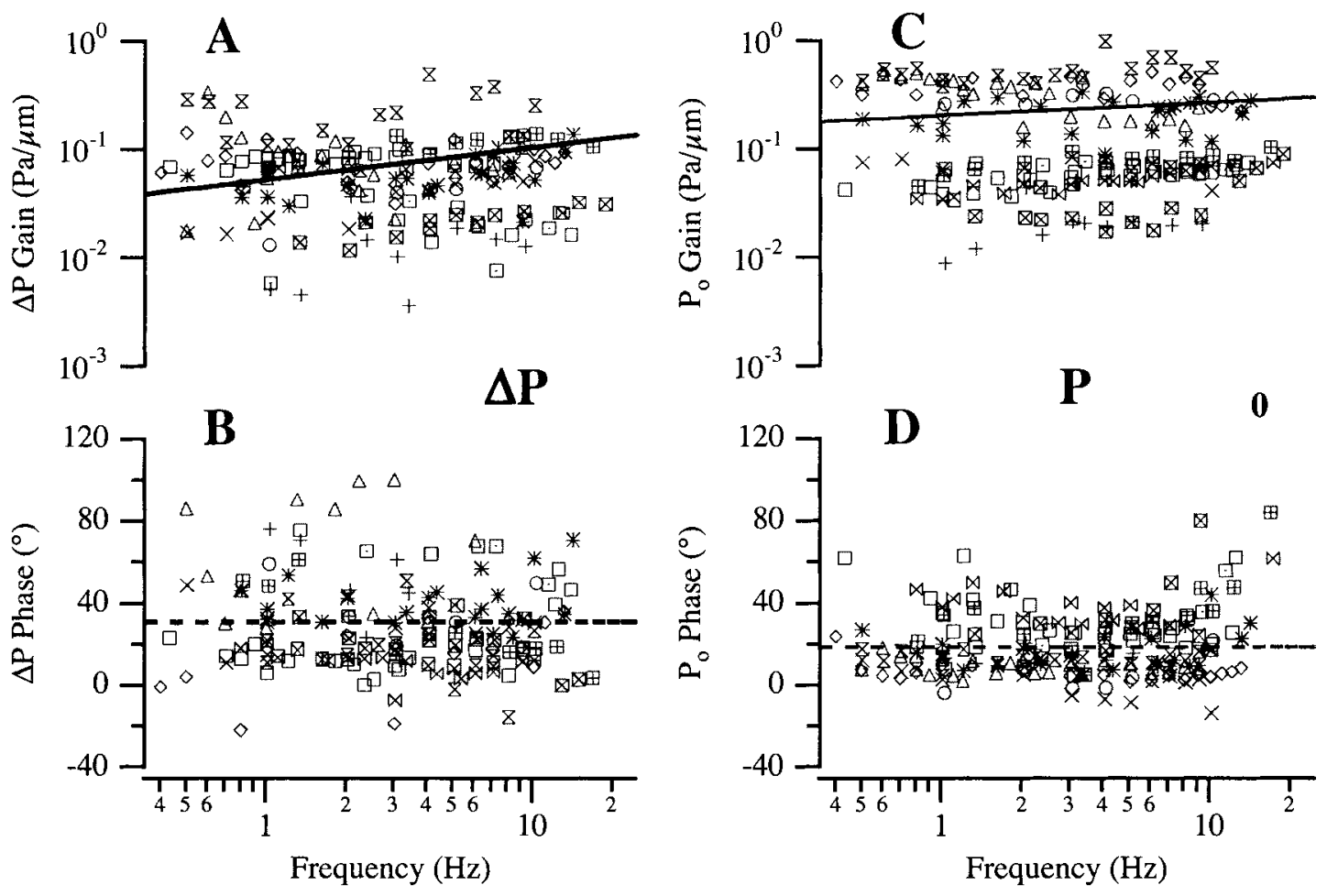

FIG. 6. Summary pressure data for 12 fish. A, B Transcupular differential pressure $(\Delta P)$ data and $\mathbf{C}, \mathbf{D}$ dilational pressure $\left(P_{0}\right)$ data in response to mechanical indentation of the canal duct $(\mu \mathrm{m})$. Results are plotted in Bode form: gain $(\mathrm{Pa} / \mu \mathrm{m})$ and phase (deg re: peak indent) as functions of frequency $(\mathrm{Hz})$. Individual markers denote data from

different fish. Phase outliers occurred during some low-gain responses. Solid lines show best-estimate fractional-power transfer functions for the population based on averaging least-squares parameters for all animals tested (see Table 1).

by viscosity (Abnet and Freeman 2000; Malvern 1969; Fung 1981). $\Delta$ Parises from the resistance of the cupula to mechanical displacement; therefore, the gain exponent of $m_{\Delta P}=0.33$ and phase of $29^{\circ}$ indicate that the volumetric impedance of the cupula exhibited both viscous and elastic properties (viscoelastic). The fact that the phase of $\Delta P$ was relatively constant over a wide range of frequencies and that the gain exponent was fractional corresponds to a material with a broad relaxation spectrum typical of soft hydrated biological tissues and gels (Fung 1981, 1990).

The dilational pressure $P_{0}$ is the average endolymph pressure within the ampulla and would be zero if and only if $P_{\mathrm{HC}}$ and $P_{\mathrm{U}}$ had equal magnitudes but opposite signs. This was not the case in the present experiments nor is it expected to be the case during physiological head rotation (Rabbitt et al. 1999). The example $P_{0}$ gain shown in Figure 5 (animal B) was nearly constant with frequency yielding a gain exponent close to zero $\left(m_{P_{0}} 0\right)$. The phase of $P_{0}$ averaged $0.5^{\circ}$. Similar to $\Delta P$ discussed above, the average phase $\bar{\theta}$ exhibited a close correlation with the optimized transfer function phase $\theta_{P_{0}}=m_{P_{0}} \pi / 2$, yielding a correlation coefficient of $R=$ 0.97 for the population of animals. Since $P_{0}$ arises from the resistance of the labyrinth to volumetric expansion, the low gain exponent and nearly zero phase indicate

that the volumetric impedance of the membranous labyrinth was dominated by stiffness (i.e., membranous duct elasticity).

Figure 6 summarizes the transcupular and dilational pressures measured in 12 animals yielding a total of 209 pressure data points. The number of frequencies tested in each animal ranged from a minimum of 6 to a maximum of 28. Transcupular pressure data are shown as Bode plots in the left panels $(\Delta P$ : A, gain; $B$, phase re: peak indent) and dilational pressure data are shown as Bode plots in the right panels $\left(P_{0}\right.$ : C, gain; D, phase re: peak indent). Individual markers denote different animals. The transcupular pressure $(\Delta P)$ gain averaged $0.059 \mathrm{~Pa} / \mu \mathrm{m}$ (mode $=0.058 \mathrm{~Pa} /$ $\mu \mathrm{m}$ ), and the phase advance averaged $29.4^{\circ}$ (mode $\left.=10.8^{\circ}\right)$. Using the established relationship between indentation and head rotation of $4^{\circ} / \mathrm{s}$ head rotation per $\mu \mathrm{m}$ in the toadfish gives an average $\Delta P$ gain of $0.015 \mathrm{~Pa}-\mathrm{s} / \mathrm{deg}$ (phase of $\Delta P$ is the same relative to peak indentation or peak angular head velocity; see Rabbitt et al. 1995). The gain exponent was computed separately for each animal and subsequently averaged over the population to define the average gain exponent for $\Delta P$ of $m_{\Delta P}=0.29( \pm 0.067 \mathrm{avg}$. SD). The average gain of the transfer function is shown as the 
solid line in Figure 6A and the corresponding phase is shown as a dashed line in Figure 6B (also see Table 1).

The dilational pressure $\left(P_{0}\right)$ gain averaged $0.12 \mathrm{~Pa} /$ $\mu \mathrm{m}$ (mode $=0.063 \mathrm{~Pa} / \mu \mathrm{m})$ and the phase advance averaged $21.8^{\circ}\left(\right.$ mode $\left.=3.6^{\circ}\right)$. This can be converted to pressure per unit volume of fluid injection using $0.6 \mathrm{~nL} / \mu \mathrm{m}$ to obtain the average dilational pressure gain of $0.20 \mathrm{~Pa} / \mathrm{nL}$ (see Rabbitt et al. 1995). The gain exponent was computed separately for each animal and used to compute the population average $m_{P_{0}}=$ 0.17 ( \pm 0.037 avg. SD). The average transfer function is shown in Figure 6 in the form of gain (C, solid) and phase (D, dashed). The low gain exponent and low phase indicate that $P_{0}$ was dominated by elasticity of the membranous labyrinth and had only a small viscous contribution. While $P_{0}$ was nearly constant across all frequencies tested for a given fish, it was found to vary considerably in magnitude between individual fish.

\section{Afferent nerve sensitivity to $\Delta P$}

Semicircular canal afferents in the oyster toadfish show a wide range of sensitivities and phase responses to sinusoidal mechanical indentation and/or angular head rotation (Boyle and Highstein 1990; Rabbitt et al. 1996). For a $2 \mathrm{~Hz}$ mechanical indentation of the HC duct, for example, a typical low-gain afferent might have a gain of $5 \mathrm{imp} / \mathrm{s}$ per $\mu \mathrm{m}$ and a phase advance of $10^{\circ}$, while a more sensitive high-gain afferent might have a gain of $80 \mathrm{imp} / \mathrm{s}$ per $\mu \mathrm{m}$ and a phase advance of $80^{\circ}$. Present data show that the transcupular pressure responsible for displacing the cupula and generating these afferent responses is $0.05 \mathrm{~Pa} / \mu \mathrm{m}$. This level of $\Delta P$ pressure corresponds to a $2 \mathrm{~Hz}$ threshold of sensitivity ${ }^{1}$ of $1 \mathrm{imp} / \mathrm{s}$ per $10^{-3} \mathrm{~Pa}$ for the sensitive afferents and $10^{-2} \mathrm{~Pa}$ for less sensitive afferents.

Since individual afferents vary their firing modulation as a function of stimulus frequency, the threshold of a particular afferent to transcupular pressure also changes with frequency. The frequency dependence can be roughly approximated by comparing the gain exponent of the afferent response $\left(m_{\mathrm{A}}\right)$ to the gain exponent of $\Delta P\left(m_{\Delta P} 0.29\right)$. Afferent gain exponents in the toadfish vary with stimulus frequency and range from approximately $m_{\mathrm{A}}=0$ to $m_{\mathrm{A}}=1$ Rabbitt et al. 1996, 1999; Boyle and Highstein 1990). The exponent $m_{\mathrm{A}}=0$ corresponds to low-gain velocity-sensitive afferents (LG type), while the exponent $m_{\mathrm{A}}=1$ corresponds to high-gain acceleration-sensitive afferents (A type). Figure $7 \mathrm{~A}$ is an example showing the response of a sensitive A-type afferent to $1.6 \mu \mathrm{m}$ sinusoidal

\footnotetext{
${ }^{1}$ The threshold level is defined here using a neural modulation of 1 impulse/s per cycle of the stimulus.
}
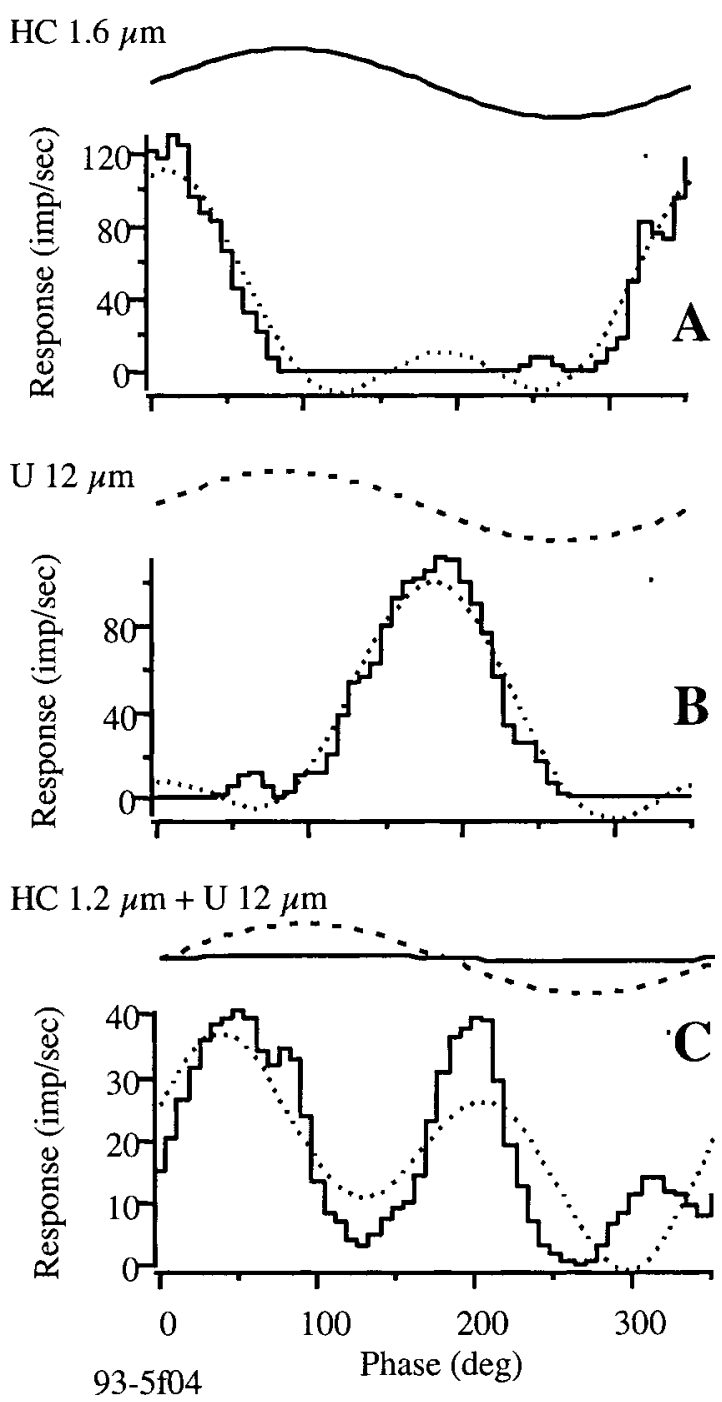

FIG. 7. Response histogram showing afferent modulation in response to $\Delta P$ and $P_{0}$. A The firing rate (impulses/s) of a sensitive afferent in response to mechanical indentation of the horizontal canal limb (solid line, $\mathrm{HC} \pm 1.6 \mu \mathrm{m}$ ) as a function of phase (bottom axis, $\mathrm{deg}$ ) in the $2 \mathrm{~Hz}$ stimulus cycle. Dotted lines in each panel show the sum of the first and second harmonics of the afferent modulation. For $\mathrm{HC}$ indentation, the peak response for this afferent was advanced $82^{\circ}$ relative to the peak indentation stimulus. B The response of the same afferent to mechanical indentation of the utricle (dashed line, $\mathrm{U} \pm 12 \mu \mathrm{m}$ ) exhibited a $90^{\circ}$ phase lag. Pressure in the ampulla during independently applied $\mathrm{HC}$ or $\mathrm{U}$ indentation $(\mathbf{A}, \mathbf{B})$ was dominated by $\Delta P$. C When the two stimuli were presented at the same time (solid $\mathrm{HC} \pm 1.2 \mu \mathrm{m}+$ dashed $U \pm 12 \mu \mathrm{m}$ ), at levels that canceled $\Delta P$ and maximized $P_{0}$, the afferent response showed a period doubling with a large second-harmonic component. The second-harmonic response demonstrates sensitivity to $P_{0}$.

indentation of the $\mathrm{HC}$ duct at $2 \mathrm{~Hz}(\Delta P 0.08 \mathrm{~Pa}$ in this case). The afferent response (impulses/s) is shown as a binned histogram (Fig. 7A, solid) along with a two-harmonic curve fit (Fig. 7A, dotted). As noted above, A-type afferents, like the one shown in Figure $7 \mathrm{~A}$, have a gain exponent of $m_{\mathrm{A}} 1$ (for Bode plots see 
Boyle and Highstein 1990; Rabbitt et al. 1995, 1996). Since the afferent gain exponent $m_{\mathrm{A}}$ is larger than the transcupular pressure exponent $m_{\Delta P}, \Delta P$ sensitivity of A-type afferents increases with increasing frequency (approximately 5 times more sensitive at $20 \mathrm{~Hz}$ re: the $2 \mathrm{~Hz}$ baseline of $10^{-3} \mathrm{~Pa}$ ). It should be noted that this simple approximation uses a power-law relationship between gain and frequency giving a straight line on a $\log -\log$ Bode plot. A more accurate analysis would need to use the frequency response for the specific afferent and for $\Delta P$ in the specific animal.

\section{Afferent nerve sensitivity to $P_{0}$}

Neural responses to the transcupular pressure described above were expected since $\Delta P$ is the force per unit area driving transverse displacement of the cupula. To investigate whether or not dilational pressure $\left(P_{0}\right)$ could also influence afferent nerve discharge, we combined mechanical indentation of the HC duct with indentation of the utricular vestibule to control the relative contributions of $\Delta P$ and $P_{0}$ (Fig. 1 , HCI and UI). Mechanical indentation of the U generated $\Delta P$ and $P_{0}$ similar to indentation of the HC duct but the phase of $\Delta P$ was reversed (Rabbitt et al. 1995). In addition to the reversal in phase, the large size of the utricular vestibule caused $\Delta P$ to be reduced and $P_{0}$ to be increased for the pressures generated by HC indentation (Rabbitt et al. 1995). Application of HC indentation and $\mathrm{U}$ indentation at the same time allowed us to minimize $\Delta P$ and maximize $P_{0}$ by adjusting the magnitude and phase of the two stimuli to eliminate (or minimize) the first-harmonic afferent modulation. This balanced stimulus generated nearly equal pressures on both sides of the cupula such that $P_{0} P_{\mathrm{HC}} P_{\mathrm{U}}$ and $\Delta P 0$. As reported previously (Rabbitt et al. 1994), many low-gain afferent nerves did not show any detectable response modulation to this balanced stimulus while, unexpectedly, some HG- and Atype afferents exhibited modulation in the absence of $\Delta P$. These units were termed $P_{0}$-sensitive.

The response of a typical $P_{0}$-sensitive afferent is shown in Figure 7 in the form of phase histograms. As noted above, the top panel (A) shows the response to a sinusoidal $\pm 1.6 \mu \mathrm{m}$ indentation of the HC duct at $1 \mathrm{~Hz}$. The pressure acting on the cupula in this $\mathrm{HC}$ indentation case was dominated by sinusoidal $\Delta P(0.1$ $\mathrm{Pa}$ ) and resulted in afferent modulations nearly equivalent to that elicited by $6.4^{\circ} \mathrm{s}$ angular head velocity (Rabbitt et al. 1995). The peak in the phase histogram for $\mathrm{HC}$ indentation (Fig. 7A) occurred $82^{\circ}$ advanced from the peak indentation stimulus (solid line). Figure $7 \mathrm{~B}$ shows the response of the same afferent to a \pm 12 $\mu \mathrm{m}$ indentation of the utricular vestibule (long dashed line). The pressure acting on the cupula in the case of $\mathrm{U}$ indentation was also dominated by $\Delta P$, but the phase was reversed relative to $\mathrm{HC}$ indentation because the stimulus was on the opposite side of the cupula. The peak afferent modulation for $\mathrm{U}$ indentation lagged the peak indentation by $90^{\circ}$ and was reversed by $172^{\circ}$ with respect to the response for HC duct stimulation. When $\mathrm{HC}$ and $\mathrm{U}$ indentations were applied at the same time and adjusted to minimize the firstharmonic afferent modulation [Fig. 7C, $1.2 \mu \mathrm{m} \mathrm{HC}$ (solid-line) $+12 \mu \mathrm{m}$ utricle (dashed line) ], $\Delta P$ was minimized and the stimulus became a dilational pressure acting on both sides of the cupula $\left(P_{0} 1.6 \mathrm{~Pa}\right.$ in this case dominated by $\mathrm{U}$ indentation). The afferent response during $P_{0}$ stimulation was not canceled but instead showed rectification (Fig. 7C). This particular afferent responded just as robustly but at two distinct phases relative to the sinusoidal stimulus, thus generating a large second-harmonic component. The first peak was phase advanced to the positive peak in the sinusoidal $P_{0}$ waveform, while the second peak was phase advanced to the negative peak in the sinusoidal $P_{0}$ waveform. Dotted lines in each panel show the addition of the first- and second-harmonic components of the phase histograms. For this afferent, the amplitude of the second harmonic during $P_{0}$ stimulation was approximately half the value of the first harmonic during $\Delta P$ stimulation, resulting in nearly the same number of modulated neural impulses per cycle for both $P_{0}$ and $\Delta P$ stimuli. Since $P_{0}$ during the balanced stimulus was 20 times larger than $\Delta P$ during $\mathrm{HC}$ indent alone, sensitivity of the afferent to $P_{0}$ was less than that to $\Delta P$.

To illustrate more clearly the balancing of the HC and $\mathrm{U}$ indentation stimuli, the response of a $P_{0}$-sensitive afferent is shown in Figure 8 as a function of $\mathrm{HC}$ indentation amplitude while a constant $\mathrm{U}$ indentation amplitude was maintained. HC and $\mathrm{U}$ stimulators were locked in phase with each other at $2 \mathrm{~Hz}$. HC duct indentation was gradually increased in discrete steps from zero to a magnitude that minimized the firstharmonic afferent response generated by the constantmagnitude $\mathrm{U}$ indentation (nondimensional ratio $\mathrm{HC}$ / $\mathrm{U}=0.5)$ and then further increased to twice the center value $(\mathrm{HC} / \mathrm{U}=1)$. Low-gain afferents, which responded only to $\Delta P$-induced displacement of the cupula, responded with zero gain when the two indentation stimuli were balanced (see Rabbitt et al. 1994). In contrast, HG- and A-type afferents, including the A-type afferent shown in Figure 8A, had a residual first harmonic that could not be completely eliminated by adjusting the $\mathrm{HC}$ stimulus. In addition, the firstharmonic phase (B) showed a more gradual transition of $180^{\circ}$ rather than a sharp jump that would be expected for an afferent that was responsive only to $\Delta P$. Sensitivity to a combination of $\Delta P$ and $P_{0}$ quantitatively accounts for this response (Rabbitt et al. 1994).

While the first harmonic is suggestive, the second 

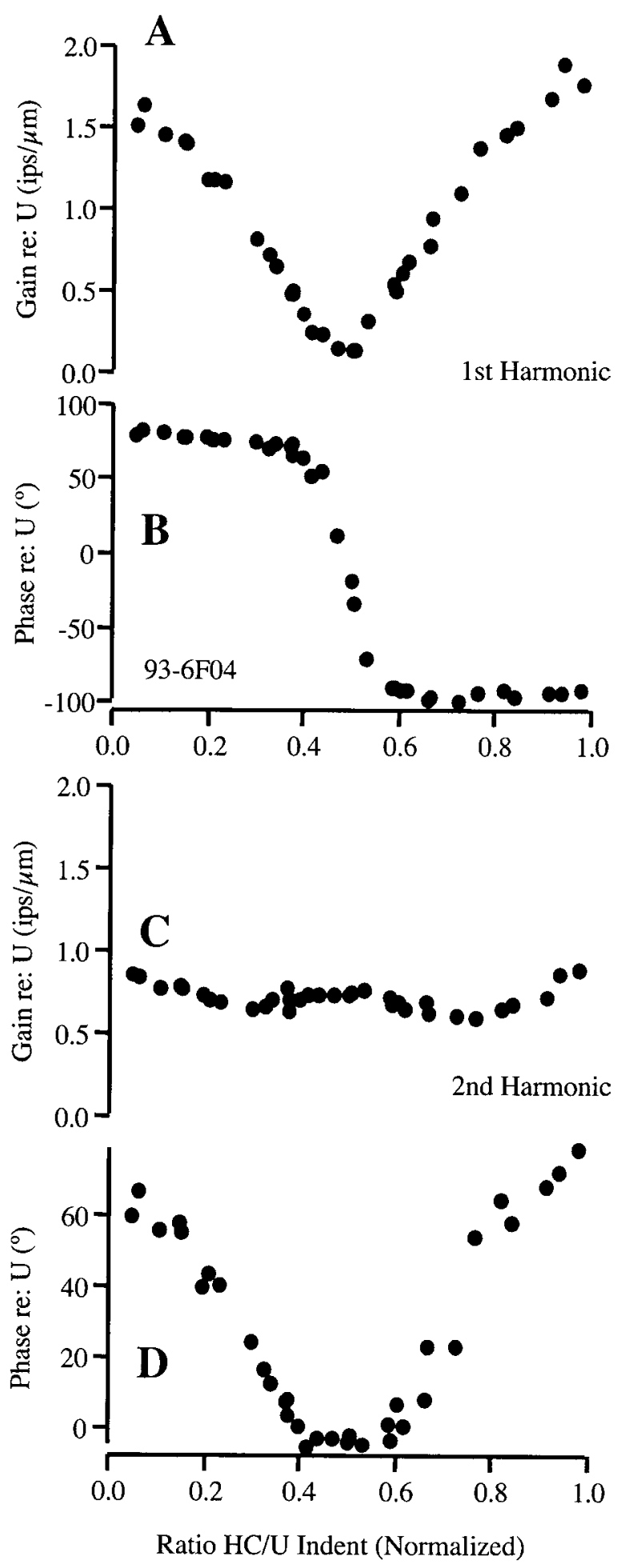

FIG. 8. First- and second-harmonic responses for a $P_{0}$-sensitive afferent. First- and second-harmonic gain (impulses/s per $\mu \mathrm{m}$ indent) and phase (deg re: indent) are shown as functions of the nondimensionalized ratio of horizontal canal $(\mathrm{HC})$ indent to utricle $(U)$ indent (see Fig. 7). When the two stimuli were balanced to maximize $P_{0}$ and minimize $\Delta P$, the first-harmonic gain (A) was minimized but the second-harmonic gain $(\mathbf{C})$ continued to show a robust response modulation. harmonic, shown in Figures 8C and D, is more revealing. Notice that the second harmonic was quite large, even when the stimulus was dominated by $P_{0}(\mathrm{HC} /$ $\mathrm{U}=0.5)$. This corresponds to the response type shown in Figure $7 \mathrm{C}$ where a rectified phase histogram was observed - the afferent had an excitatory response for positive $P_{0}$ and a nearly identical excitatory response for negative $P_{0}$. The origin of a large rectified response is related to distension of the ampulla and cupular deformation, as discussed below. It is important to note that the same result is obtained over a broad range of stimuli and, therefore, is not the result of an amplitude-dependent nonlinearity.

To quantify $P_{0}$ sensitivity, the first- and second-harmonic modulations of individual afferents were determined for the balanced $P_{0}$ stimuli at $2 \mathrm{~Hz}$. After the two indenters were balanced to minimize the first harmonic, the phase of the HC duct stimulator was then reversed to generate a $\Delta P$-dominated stimulus and the first-and second-harmonic responses were again determined. Afferent sensitivity to $P_{0}$ re: $\Delta P$ was defined as the total response of each individual afferent to the $P_{0}$ stimulus divided by its response to the $\Delta P$ stimulus. Figure 9 shows the $P_{0}$ sensitivity of 32 individual afferent fibers plotted against the phase advance during $\Delta P$ stimulation. A sensitivity of " 0 " indicates the afferent did not modulate in response to the $P_{0}$-dominated stimulus, while a sensitivity of " 1 " indicates the afferent responded just as robustly to the $P_{0}$ stimulus as it did to the $\Delta P$ stimulus. The dashed line in Figure 9 shows a simple linear regression of the data showing a weak correlation with absolute phase (slope $=0.007 / \mathrm{deg}$, Pearson's $R=0.58$ ). There was no significant correlation of $P_{0}$ sensitivity with average firing rate, afferent gain, or afferent regularity (Pearson's $R<0.1$ in all cases). Note that there is a striking absence of points above the diagonal line (solid line that passes through the origin and through 1 at $90^{\circ}$ phase advance). Therefore, phase advance is necessary, but not sufficient, for an afferent to show sensitivity to $P_{0}$ stimuli.

\section{DISCUSSION}

\section{Transcupular pressure $\Delta P$}

Present data show that sinusoidal mechanical indentation of the HC duct generates an average transcupular pressure of $0.05 \mathrm{~Pa} / \mu \mathrm{m}$ or, for sinusoidal head rotation, $0.013 \mathrm{~Pa}-\mathrm{s} / \mathrm{deg}$ (average $=0.4-20 \mathrm{~Hz}$ ). We constructed a simple finite element (FE) model to investigate how this transcupular pressure might be related to cupular deformations and hair bundle deflections. Figure 10 shows predictions of the FE model for cupular deformation and hair bundle 


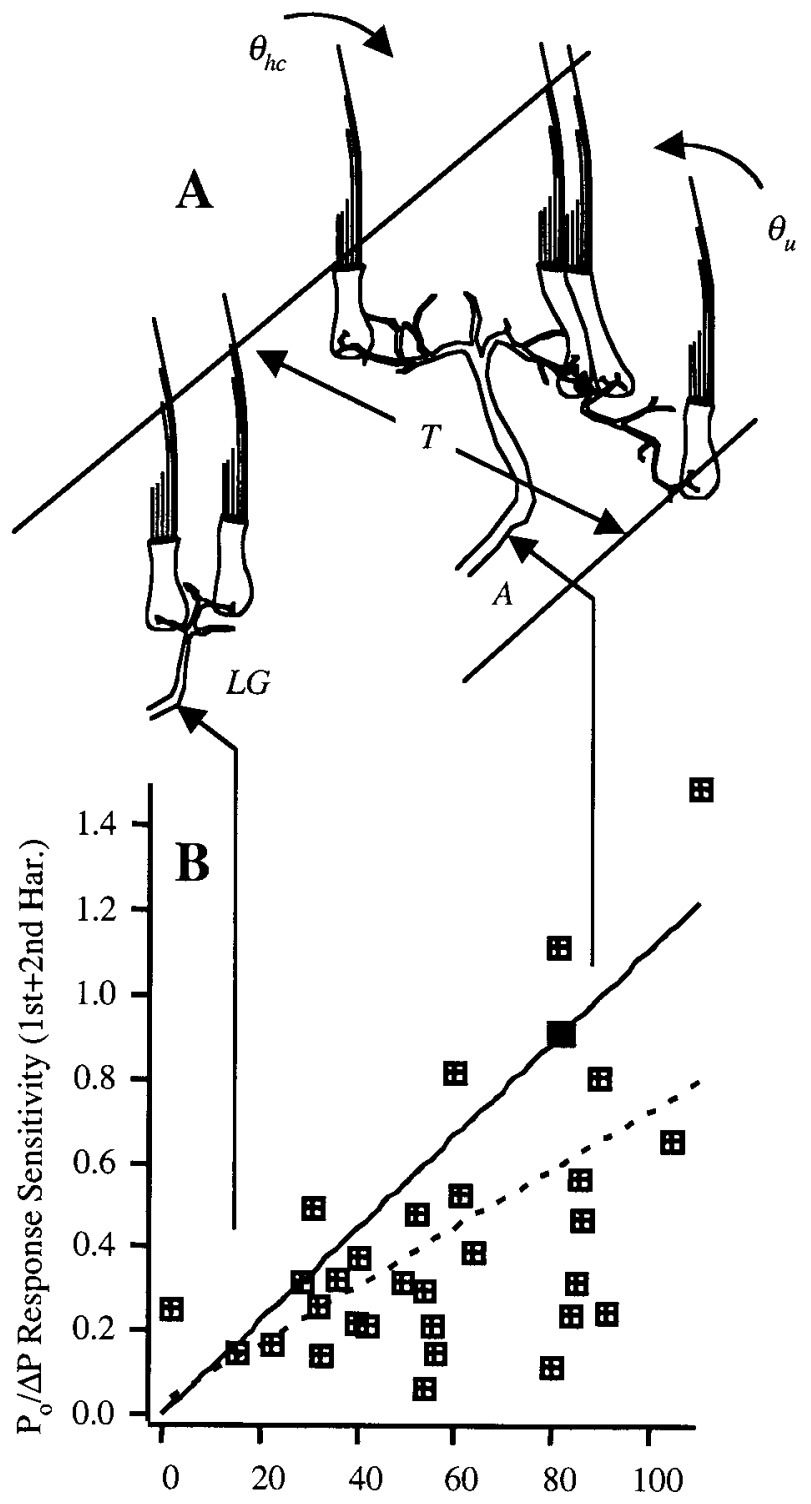

Afferent Phase Advance re: HC Indent $\left({ }^{\circ}\right)$

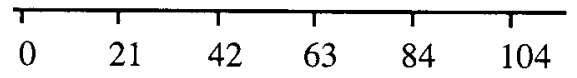

Estimated Transverse Axis (\%)

FIG. 9. A Morphological correlate of $P_{0}$ sensitivity. The amplitude of the afferent response to $P_{0}$ is characterized by the addition of the first- and second-harmonic gains measured during balanced $\mathrm{HC}+$ $\mathrm{U}$ indentation normalized by the same values obtained during $\mathrm{HC}$ indentation alone (left). B Results are plotted against the afferent phase advance re: $\mathrm{HC}$ only indent at $2 \mathrm{~Hz}$. Markers are for individual afferents, data which span the normal range of phase advances observed in this species at $2 \mathrm{~Hz}$ (Boyle and Highstein 1990). The solid line is from the origin to a value of 1 at $90^{\circ}$, and the dashed line is a linear regression of the data. The lower horizontal axis provides the approximate transverse width of the dendritic field across the crista (\% transverse distance $T$ ). Schematics in $\mathbf{A}$ indicate the extent of the dendritic tree for two example afferents labeled LG and A. These sketches are based on morphological studies of Boyle et al. (1991). Arrows pointing to the afferents denote the phase advance relative to the peak of the sinusoidal stimulus for the LG and A afferents.

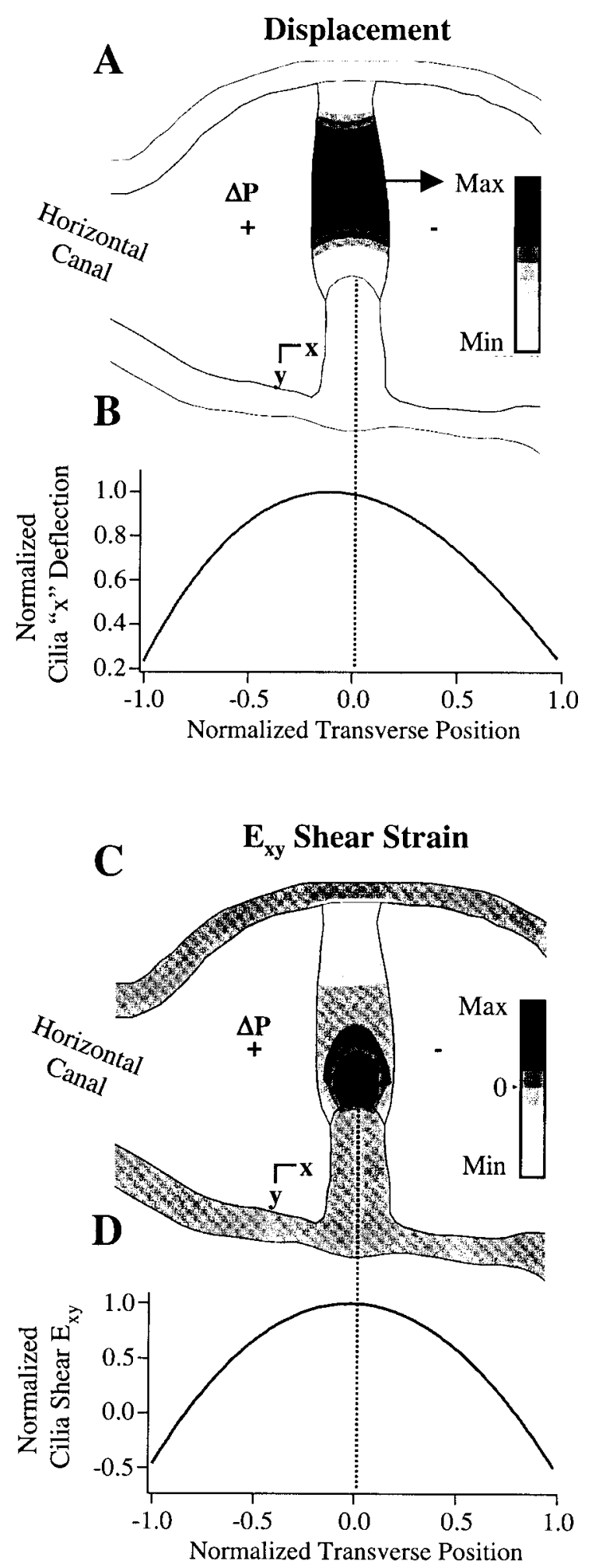

FIG. 10. FE model predictions for a pure transcupular pressure $\Delta P$ load. A Displacement and $\mathbf{C}$ shear strain of the cupula are shown. B Transverse distributions of the displacement and $\mathbf{D}$ shear strain in the cupula $-100 \mu \mathrm{m}$ above the crista, near the tips of hair cell stereocilia. 
deflection under pure transcupular $\Delta P$ pressure loading. The geometry was based on morphological data from Silver et al. (1998) and Ghanem et al. (1998). Details of the construction and implementation of the FE model are given in the Appendix. Results are shown on a cutting plane passing through the center of the ampulla and central pillar of the cupula to illustrate deformation through the transverse thickness of the cupula and near the surface of the sensory epithelium. Transverse cupular displacement is shown as a grayscale contour plot for positive $\Delta P$ (Fig. 10A); white corresponds to zero displacement and black corresponds to maximum displacement. The largest displacement was predicted to be at a point approximately halfway between the sensory epithelium and the ampullary apex, consistent with the diaphragm displacement mode reported by Hillman and McLaren (1979). The solid curve (Fig. 10B) shows the predicted hair bundle deflection as a function of transverse position across the crista ( $x$ coordinate). The normalized transverse axis ranges from -1 on the canal lumen side of the crista to +1 on the utricular side of the crista. The largest $x$ component of deflection of cilia was predicted to be approximately halfway through the thickness of the cupula. Deflection itself, however, is not the main mechanical stimulus causing stretch of the stereociliary crosslinks. Because of the diagonal orientation of crosslinks between stereocilia, stretch of the links is more closely related to transverse shear strain than to displacement. Figure $10 \mathrm{C}$ is a grayscale plot showing that the largest shear strain was predicted to act at the location of the stereocilia near the surface of the sensory epithelium, not at the center of the cupula. The graph in Figure 10D is a prediction for the distribution of $x y$ shear strain acting on the hair bundles as a function of position through the thickness of the cupula. Activation of hair cells was predicted to be a maximum at the attachment of the cupula to the crista about halfway through the transverse thickness. This demonstrates how the configuration of the cupula serves to effectively convert $\Delta P$ and cupular displacements into a signal appropriate to activate hair cell transduction channels. FE model results provide the spatial configuration of the FE model cupula in response to a static transverse pressure $\Delta P$ and, hence, they are expected to provide a qualitative estimate of actual cupular deformation for low-frequency head movements $(<2 \mathrm{~Hz})$. At higher frequencies, inertia would be expected to influence the deflected shape (Damiano and Rabbitt 1996). We have also neglected the inhomogeneous, anisotropic structure of the cupula which would be expected to influence the detailed spatial distribution of shear strain across the surface of the crista.

It was suggested in a previous report that $\Delta P$ might have a frequency response (gain exponent and phase) that closely aligned with angular acceleration of the head or velocity of indentation and, thus, might correlate with the phase advance and gain exponents of HG- and A-type afferents (Rabbitt et al. 1994). Present results show that the gain exponent $\left(m_{\Delta P}\right)$ and phase advance $\left(\theta_{\Delta P}\right)$ of $\Delta P$ are both relatively low compared with $m_{\mathrm{A}}$ and $\theta_{\mathrm{A}}$ of A-type afferents. Therefore, the frequency response of $\Delta P$ does not provide a direct correlate to the neuraL frequency response and cannot explain the dynamics of highly phase-advanced afferent types as previously hypothesized. Rather, the relatively low $m_{\Delta P}$ and $\theta_{\Delta P}$ observed here provide additional evidence that canal macromechanics accounts for only a small fraction of the high-frequency enhancements in gain and phase reflected in the responses of high-gain afferents (Goldberg and Fernández 1971a, b; Damiano and Rabbitt 1996; Highstein et al. 1996; Rabbitt et al. 1999).

The transcupular pressure $\Delta P$ reported above can be combined with the cupular volume displacement $(Q)$ to estimate the cupular volumetric impedance $\left(Z_{c}=\Delta P / Q\right)$. The frequency dependence of $Z_{c}$ characterizes the viscoelastic mechanical properties of the cupula and any amplitude dependence characterizes nonlinearity of cupular responses. Although $Q$ was not measured in the present experiments, theoretical and experimental studies indicate that $Q$ is proportional to and in phase with angular velocity of the head (or HC duct indentation), at least in the midfrequency band studied here (Steinhausen 1933; Oman et al. 1987; VanBuskirk 1987; Rabbitt et al. 1995; Damiano and Rabbitt 1996; Rabbitt 1999; Yamauchi et al. 2000). We saw no evidence of amplitude-dependent nonlinearity under the conditions studied. Therefore, $Z_{c}$ is expected to be proportional to $\Delta P$. The phase $\left(\theta_{\Delta P}\right)$ and gain exponent $\left(m_{\Delta P}\right)$ indicate that the impedance of the cupula has a form typical of soft tissues and gels (Fung 1981, 1990; also see Figs. 5 and 6). Viscoelastic materials of this type are well characterized by continuous-spectrum, quasilinear, viscoelastic models. Simple discrete-spectrum Kelvin-Voigt-type viscoelastic models, applied previously to the cupula, do not predict the observed $\Delta P$ frequency response and hence do not capture the viscoelastic properties of the cupula (Damiano and Rabbitt 1996; Rabbitt et al. 1999). It should be noted, however, that cupular volume displacements predicted by Kelvin-Voigt models are insensitive to cupular viscosity and therefore may be adequate for some macromechanical investigations.

\section{Active hair bundle movement}

There is a solid body of evidence that indicates the existence of electromotility of stereocilia in isolated vestibular hair cells, including otolith and semicircular canal hair cells (Howard and Hudspeth 1987; Assad 
and Corey 1992; Rüsch and Thurm 1990; Ricci et al. 2000; Denk and Webb 1992; Benser et al. 1996; Martin and Hudspeth 1999). It is not yet known if this process has a role in reverse transduction and amplification of cupular displacements in the semicircular canals. If true, one might expect to observe an amplitudedependent nonlinearity in the cupular volumetric impedance. Such an effect was not observed under conditions of the present experiments. If an active component were present, it was overpowered by passive mechanics and not identifiable under the conditions tested.

\section{Dilational pressure $P_{0}$}

In addition to sensitivity to $\Delta P$, present data also show that neural responses to dilational pressure occur. Sinusoidal mechanical indentation of the HC duct and utricular vestibule a $2 \mathrm{~Hz}$ revealed a sensitivity of $P_{0} 5$ $\times 10^{-2} \mathrm{~Pa}$ which generated a threshold firing rate modulation $1 \mathrm{imp} / \mathrm{s}$ in sensitive afferents. Most $P_{0^{-}}$ sensitive afferents responded in a highly rectified manner (Figs. 7 and 8). Results of the finite element model, shown in Figure 11 for a $P_{0}$ translabyrinthine pressure increase, indicate a mechanical origin of the rectified response. According to the model, increased endolymph pressure causes distension of the ampulla and leads to cupular deformation. Since the cupula is highly hydrated and nearly incompressible (Poisson ratio $=0.5$ ), distension of the ampulla (Fig. 11A) was accompanied by a compensatory decrease in cupular thickness. For positive $P_{0}$, the HC lumen side of the cupula displaced toward the utricle, while the utricular side displaced toward the HC lumen. Hair bundle deflections caused by $P_{0}$ were predicted to be zero near the center of the crista and maximum at the extreme transverse positions on the sensory epithelium (Fig. 11B). The shear strains predicted to act on the hair bundles are even more revealing (Figs. 11C and $\mathrm{D}$ ). At the center of the crista the shear strain was approximately zero, so we would not expect activation of central hair cells during $P_{0}$ stimulation. In contrast, the shear strains acting at the outermost edges of the cupula were predicted to be quite large and nearly equal in magnitude to that present during $\Delta P$ stimulation. One side of the sensory epithelium was predicted to experience negative shear strains while the other side positive shear strains. Hence, for sinusoidal $P_{0}$ stimuli, the FE model predicts that afferents having wide transverse dendritic fields would receive excitatory synaptic inputs from hair cells during both positive and negative phases of the stimulus. This, in turn, would lead to fully rectified neural responses exactly as observed in the experiments.

Results indicate that the large second-harmonic responses to $P_{0}$ arose from simultaneous excitation and inhibition of different hair cells residing within widely spaced transverse regions of the crista and innervated by single afferents. The more phaseadvanced afferents have been shown previously to exhibit wide transverse dendritic arborizations within the crista in this species (Boyle et al. 1991). These are the same afferents that exhibited the large secondharmonic responses. A schematic of the transverse extent of the dendritic tree for two afferents-A-type (acceleration) and LG-type (low gain) - is provided at the top of Figure 9. Afferents falling on the right side of Figure 9 are the high-gain and acceleration type and have wide dendritic fields and inputs from hair cells on both the canal lumen and utricular sides of the crista, whereas those on the left are the low-gain neurons and have small dendritic fields which receive input from only one small region of the crista. To emphasize this morphological correlation, we have added a second horizontal axis at the bottom of the figure that shows the percent of the transverse axis $(T)$ of the crista spanned by the dendritic field of individual afferents. This percentage axis was determined using the regression line given by Boyle et al. (1991, Fig. 15) which correlates the extent of the dendritic field with afferent phase advance (converted from rotational stimuli to mechanical indentation using Rabbitt et al. 1994). This approximation resulted in a transverse axis slightly exceeding $100 \%$ in several cases because of the use of population-average correlation coefficients. Present data show that a wide dendritic field is necessary for rectified $P_{0}$ sensitivity.

It is important to note that the second-harmonic afferent response was not the result of an amplitudedependent nonlinearity; the second harmonic was proportional to the stimulus amplitude and the gain was constant over the range tested. The appearance of the second harmonic is better described in terms of convergence of multiple inputs, each originating at different sites within the sensory epithelium. If the process were a simple summation of passive hyper-and depolarizing inputs, reflecting a periodic modulation of transmitter binding about a steady resting value, then one would expect the hyperpolarizing signal from the canal side of the crista to cancel the depolarizing signal from the utricular side of the crista. Perhaps this is true for the subset of afferents that did not exhibit the $P_{0}$ response, but clearly this is not the case for afferents exhibiting the large second-harmonic response. Rather than passive summation, the observed responses are more consistent with the hypothesis that rectified pulses are initiated within individual dendritic branches and summate to generate the total afferent response. In this view, postsynaptic channels in the individual dendrites produce nonlinear responses, which summate to generate the intra-axonal afferent response. The behavior may have 
analogy to nonlinear soliton waves (Jeffrey and Kawahara 1982). It also appears that the responses cannot be accounted for in terms of variations in electrotonic length of the various dendrites. The delay time from the hair cell activation to axonal spikes is known to range from 0.7 to $1.1 \mathrm{~ms}$ in this species (Highstein et al. 1996; Rabbitt et al. 1995). Most of this delay is synaptic in origin, indicating that variations in the delay associated with the electronic length of individual afferents and associated dendritic arborizations is small. This being the case, the signals converging from the individual dendrites must be rectified as the result of postsynaptic biophysics prior to summation.

In view of afferent responses to $P_{0}$ morphological projections of responding afferents into the crista, and FE model predictions of the cupular deformation field, we conclude that ampullary distension and cupular deformation underly the observed afferent responses to changes in translabryinthine dilational pressure $\left(P_{0}\right)$.

\section{Adaptation to maintained $P_{0}$ pressure}

Present experiments were limited to dynamic stimuli $(0.4-20 \mathrm{~Hz})$ and therefore do not directly address afferent firing-rate responses to maintained DC pressures. The stiffness-dominated volumetric impedance of the system, however, does indicate a capacity for the membranous labyrinth to maintain translabyrinthine pressures over extended periods of time. Maintained endolymph dilational pressure $\left(P_{0}\right)$ would generate prolonged cupular deformations and associated hair bundle displacements (Fig. 11). Over extended periods of increased endolymph pressure, adaptation would be expected to reset the operating point and return afferent nerve discharge to prestimulus levels. Therefore, the time course of adaptation would dominate neural responses for maintained stimuli. Afferent nerves in the toadfish show a wide range of adaptation time constants for maintained cupular displacements ranging from tens of milliseconds to tens of minutes (Rabbitt et al. 2000). It is reasonable to expect adaptation to maintained pressure stimuli to follow the same time course. Low-gain, velocity-sensitive, afferent nerves in the toadfish exhibit the slowest adaptation. These units are analogous to the regularly discharging, velocity-sensitive, afferent nerves in primates (Fernández and Goldberg 1971; Goldberg and Fernández $1971 \mathrm{a}, \mathrm{b})$-neurons that provide the primary input to the vestibulo-ocular reflex system (Minor and Goldberg 1991). Therefore, one would expect a maintained change in endolymph pressure to result in a compensatory vestibulo-ocular response followed by a period of relatively slow adaptation. Direct experimental data testing this hypothesis are not yet available.
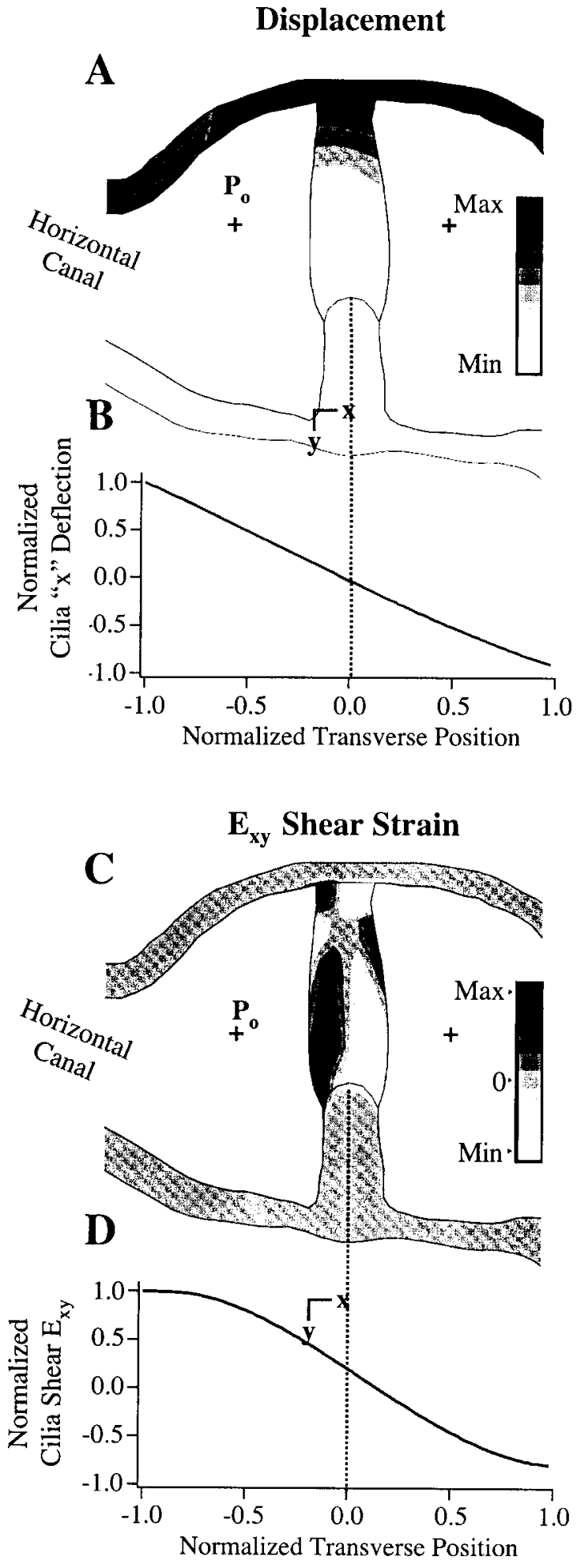

FIG. 11. FE model predictions for pure dilational pressure $P_{0}$ load. Notation same as in Figure 10.

\section{Influence of perilymphatic pressure}

Given the present endolymph pressure data, it is natural to question whether changes in perilymphatic pressure could also elicit $P_{0}$-sensitive responses. Rather 
large changes in perilymphatic pressure occur in response to changes in posture, blood pressure, cerebrospinal fluid pressure, ambient pressure, gravity, and numerous pathological conditions (Carlborg et al. 1990; Carlborg and Farmer 1983; Kerth and Allen 1963; Kishimoto et al. 1983; Konradosson et al. 1994; Parker 1977). In the present experiments the perilymphatic space was opened widely so that its pressure always equilibrated with the ambient value in the experimental chamber. Therefore, modulation of the ambient pressure induced direct modulation of the perilymphatic pressure. Semicircular canal afferents did not respond when we manipulated the ambient pressure and perilymphatic pressure. This is not surprising since fish swim at various depths in waters with ambient pressures spanning several atmospheres. These natural ambient pressure fluctuations are at least $10^{6}$ times higher than the translabyrinthine $P_{0}$ pressures shown here to induce afferent responses. Results are consistent with the findings of Salt and DeMott (1998) that show cochlear insensitivity to perilymph injections in scala tympani. Hence, the inner ear appears to be designed to accurately match the endolymphatic pressure with unavoidable changes in perilymphatic pressure. This minimizes sensitivity to ambient pressure changes and is facilitated in the vestibular organs by complete immersion of the membranous ducts and vestibules in a continuous perilymphatic enclosure.

\section{Data limitations}

Although the gain and phase data were highly consistent within individual animals, there were significant differences in both $P_{0}$ and $\Delta P$ recorded between different animals. Interanimal differences are reflected in the relatively wide range of fractional-power exponents and gains reported in Table 1. Part of this variation can be accounted for by the stimulus, which is known to introduce interanimal variability with changes in the location of the mechanical indenter, geometry of the labyrinth, and canal preload (Dickman and Corriea 1989a, b; Rabbitt et al. 1995). These factors may account for as much as half of the interanimal variability in the present pressure data. The remaining variability is not surprising given the expected interanimal differences in tissue mechanical properties and labyrinthine morphology. It is well established that mechanical properties of soft tissues vary considerably between individual animals with factors such as age, weight, diet, and pathological conditions (Fung 1981, 1990). Such factors were not controlled in the present experiments and could easily account for a significant fraction of the interanimal differences in pressure responses. In addition to differences in tissue mechanical properties, there are significant differences in labyrinth morphology between individual toadfish (Ghanem et al. 1998) that would also contribute to interanimal variability in the pressure data.

The linear transfer functions reported in Table 1 are strictly empirical in nature and have a mathematical form selected to be consistent with the data yet be as simple as possible. The transfer functions reported would not be expected to extend outside of the range of amplitudes or frequencies tested. One should not use the transfer functions to extrapolate the data in the frequency domain (outside $0.4-20 \mathrm{~Hz}$ ) or to extrapolate to higher pressure levels where the volumetric impedance of the membranous labyrinth is nonlinear (Wit et al. 2000). Caution should also be exercised in attempting to extend results to other species. For example, a smaller labyrinth would be expected to have higher volumetric impedance and therefore a higher $P_{0}$ gain multiplier (i.e., higher $\left.C_{P_{0}}\right)$. Extension of the pressure results to other species would require detailed knowledge of differences in labyrinthine morphology and tissue viscoelastic properties. The same note of caution applies to extension of neural threshold data to other species. A more compliant labyrinth (i.e., compliant tissue, large and/or thin) would be more susceptible to $P_{0}$-induced distention and, therefore, would have a lower threshold to $P_{0}$. Similarly, a more compliant cupula (i.e., compliant tissue, large and/or thin) would result in a lower threshold to $\Delta P$. Even with these notes of caution it seems reasonable to hypothesize on the basis of gross anatomical homology that similar levels of pressure sensitivity may be present in human semicircular canals.

\section{ACKNOWLEDGMENT}

This work was supported by the National Institute of Deafness and Other Communications Disorders Grant P01 DC01837.

\section{APPENDIX}

\section{FEM model construction}

A simple finite element model of the toadfish $\mathrm{HC}$ ampulla and cupula was constructed for the purpose of directing experiments and interpreting results. The model geometry was based on a combination of microscopic observation of fresh tissue and histological sections of the ampulla (Ghanem et al. 1998; Silver et al. 1998). For simplicity, the geometry was reduced to a rectangular 3D domain, and hence the model results are limited to the central region of the crista. The finite element mesh was composed of 8-node brick 


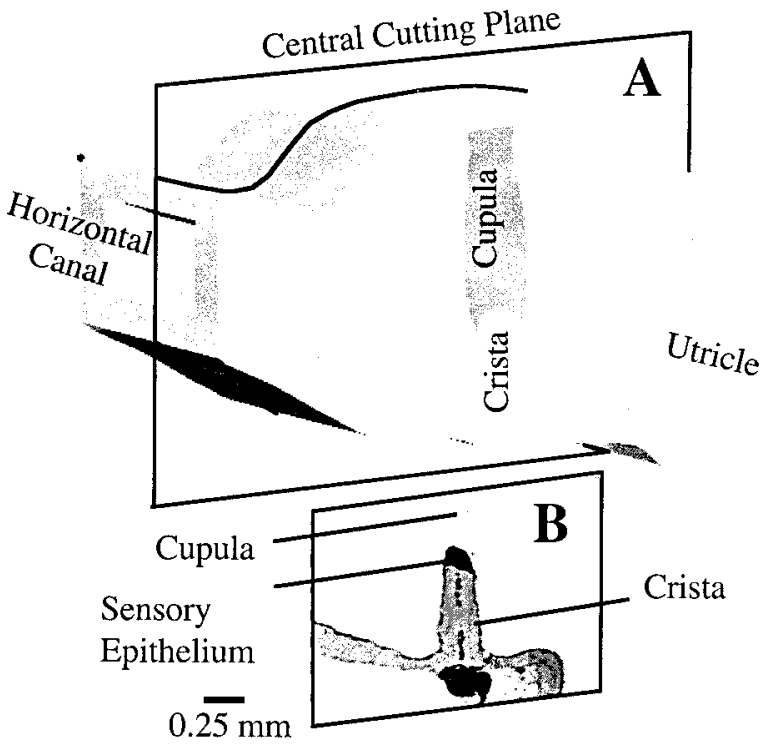

FIG. 12. FE model geometry. A Eight-node brick elements were used to construct a simplified rectangular model consisting of elastic elements for the crista and membranous labyrinth (light gray) and soft elastic elements for the cupula (dark gray). B A photomicrograph illustrating the geometry of the crista in the toadfish at the central cutting plane (Ghanem et al. 1998).

elements projected to this fish geometry, as illustrated in Figure 12A. The ampullary walls and cupula were modeled as elastic materials. The elastic moduli of the cupula (shear modulus $=0.36 \mathrm{~Pa}$, Poisson ratio $=0.5$ ) and of the membranous duct (shear modulus $=300$ $\mathrm{Pa}$, Poisson ratio $=0.5$ ) were selected to match the average cupular displacement and ampullary distension predicted by previous macromechanical models (Damiano and Rabbitt 1996; Rabbitt et al. 1999). In order to model the cupula as a totally incompressible material, an augmented Lagrangian method was used (Maker et al. 1990). The solution was constrained to a plane-strain problem. Motion of the side walls was constrained to the plane, and the bottom edge of the plane was fixed.

Two loading cases were studied: (1) transcupular pressure $\Delta P$ and (2) dilational pressure $P_{0}$. The pressure for both the $\Delta P$ and $P_{0}$ cases were applied to all endolymph wetted surfaces including the sides of the crista, the cupular leaflets, and the interior surfaces of the ampulla. The magnitude of the pressure for the $\Delta P$ case was taken to be $\Delta P=0.01 \mathrm{~Pa}$. The magnitude of pressure used in the $P_{0}$ case was taken from the present experimental measurements to be $0.12 \mathrm{~Pa}$. Since the model is linear, displacement magnitudes and shear strains scale with the elastic moduli and pressure but the spatial distribution remains constant.

The FE model presented here for the toadfish cupula is similar to the human model presented by
Njeugna et al. (1992). They modeled a two-dimensional slice of the human cupula as a thick, soft, elastic material that spanned the cross section of the ampulla and extended down the sides of a stiff elastic crista. The present model is slightly more general in that we included elasticity of the membranous duct and solved for the resulting ampullary distension. Although differences in geometry between the species cause some differences in the cupular deformation fields, the general trends for both $P_{0}$ loading and $\Delta P$ loading are similar for the two models.

\section{REFERENCES}

Abnet CC, Freeman DM. Deformations of the isolated mouse tectorial membrane produced by oscillatory forces. Hear. Res. 144:2946, 2000

Andrews JC, Bohmer A, Hoffman LF. The measurement and manipulation of intralabyrinthine pressure in experimental endolymphatic hydrops. Laryngoscope 101:661-668, 1991.

ANDREWs JC, Honrubia V. Meniere's disease. In: Baloh RW, Halmagyi GM, (eds) Disorders of the vestibular system. Oxford University Press New York, 1996, p. 300-316.

Assad JA, CoRey DP. An active motor model for adaptation by vertebrate hair cells. J. Neurosci. 12:3291-3309, 1992.

Benser ME, Marquis RE, HudsPeth AJ. Rapid, active hair bundle movements in hair cells from the bullfrog's sacculus. J. Neurosci. 16:5629-5643, 1996.

BOHMER A. Hydrostatic pressure in the inner ear fluid compartments and its effects on inner ear function. Acta Otolaryngol. (Stockh.) Suppl. 507:4-24, 1993.

Boyle R, Carey JP, Highstein SM. Morphological correlates of response dynamics and efferent stimulation in horizontal semicircular canal afferents of the toadfish, opsanus tau. J. Neurophysiol. 66:1504-1521, 1991.

Boyle R, Highstein SM. Resting discharge and response dynamics of horizontal semicircular canal afferent of the toadfish, opsanus tau. J. Neurosci. 10:1557-1569, 1990.

CAmis M. The Physiology of the Vestibular Apparatus. Clarendon Press Oxford, 1930.

CARLborg B, Farmer JC. Transmission of cerebrospinal fluid pressure via the cochlear aqueduct and endolymphatic sac. Am. J. Otolaryngol. 4:273-282, 1983.

Carlborg B, Farmer J, Carlborg A. Effects of hyperbaric pressure on the labyrinth-cochlear aqueduct patent. Acta Otolaryngol. (Stockh.) 90:386-393, 1990.

DAMiAno ER, RABbitT RD. A singular perturbation model of fluid dynamics in the vestibular semicircular canal and ampulla. J. Fluid Mech. 307:333-372, 1996.

DANCER A, FrANKE R. Intracochlear sound pressure measurements in guinea pigs. Hear. Res. 2:191-205, 1980.

Denk W, Weвв WW. Forward and reverse transduction at the limit of sensitivity studied by correlating electrical and mechanical fluctuations in frog saccular hair cells. Hear. Res. 60:89-102, 1992.

DickMAN JD, REDER PA, CORREIA MJ. A method for controlled mechanical stimulation of single semicircular canals. J. Neurosci. Meth. 25:111-119, 1988.

DiCKMAN JD, CORREIA MJ. Responses of pigeon horizontal semicircular canal afferent fibers. I. Step, trapezoid, and low-frequency sinusoid mechanical and rotational stimulation. J. Neurophysiol. 62:1090-1110, 1989a.

DICKMAN JD, CORREIA MJ. Responses of pigeon horizontal semicircular canal afferent fibers. II. High-frequency mechanical stimulation. J. Neurophysiol. 62:1102-1112, 1989b. 
EwALD JR. Physiologisch untersuchingen über das endorgan des nervus octavus. Bergmann, Wiesbaden, 1892.

Fernández C, Goldberg JM. Physiology of peripheral neurons innervating the semicircular canals of the squirrel monkey. II. Response to sinusoidal stimulation and dynamics of peripheral vestibular system. J. Neurophysiol. 34:661-676, 1971.

FUNG YC. Biomechanics: Mechanical properties of living tissues. Springer-Verlag, New York, 1981.

FuNG YC. Biomechanics: Motion, flow, stress, and growth. SpringerVerlag, New York, 1990.

Ghanem TA, Rabbitt RD, Tresco PA. Three-dimensional reconstruction of the membranous vestibular labyrinth in the toadfish, opsanus tau. Hear. Res. 124:27-43, 1998.

Goldberg JM, Fernández C. Physiology of peripheral neurons innervating semicircular canals of the squirrel monkey. I. Resting discharge and response to constant angular accelerations. J. Neurophysiol. 34:635-660, 1971a.

Goldberg JM, Fernández C. Physiology of peripheral neurons innervating semicircular canals of the squirrel monkey. II. Variations among units in their discharge properties. J. Neurophysiol. 34:676-685, 1971b.

Hess BJM, Lysakowski A, Minor LB, Angelaki DE. Central versus peripheral origin of vestibulo-ocular reflex recovery following semicircular canal plugging in the rhesus monkeys. J. Neurophysiol. 84(6):3078-3082, 2000.

HighsteIn SM, RabBitT RD, BoYLE R. Determinants of semicircular canal afferent response dynamics in the toadfish, opsanus tau. J. Neurophysiol. 75(2):575-596, 1996.

Hillman DE, MCLAREN JW. Displacement configuration of semicircular canal cupulae. Neuroscience 4:1989-2000, 1979.

HonRubia V. Pathophysiology of Ménière's disease. In: Harris JP, (ed) Ménière's disease. Kugler The Hague, Netherlands, 1999, 93-101.

HORNER KC. Functional changes associated with experimentally induced endolymphatic hydrops. Hear. Res. 68:1-18, 1993.

HOWARD I, HudSPETH AJ. Mechanical relaxation of the hair bundle mediates adaptation in mechanotransduction by the bullfrog's saccular hari cell. Proc. Natl. Acad. Sci. U S A 84:3064-3068, 1987.

Jeffrey A, Kawahara T. Asymptotic Methods in Nonlinear Wave Theory. Pitman Marshfield, MA, 1982.

KerTh JD, AlLEN GW. Comparison of the perilymphatic and cerebrospinal fluid pressures. Arch. Otolaryngol. 77:581-585, 1963.

Kishimoto S, Nagahara K, Fisch U, Dillier N. Inner ear pressure measurements: Effects of obstruction of the cochlear aqueduct and endolymphatic duct on the perilymphatic pressure. Otolaryngol. Clin. North Am. 16(1):21-35, 1983.

Konradosson KS, Carlborg AH, Farmer JC, Carlborg BIR. Perilymph pressure during hypobaric conditions-cochlear aqueduct obstructed. Acta Otolaryngol. (Stockh.) 114:24-29, 1994.

Lynch TJ, Nedzelnitsky V, Peake WT. Input impedance of the cochlea in cat. J. Acoust. Soc. Am. 72:108-130, 1982.

Maker BN, Ferencz RM, Hallquist JO (1990) Nike3d: A nonlinear, implicit, three-dimensional finite element code for solid and structural mechanics. Lawrence Livermore Nat. Lab. Tech. Rep.

MALVERN LE. Introduction to the mechanics of a continuous medium. Prentice-Hall Englewood Cliffs, NJ, 1969.

Martin P, HudsPETH AJ. Active hair bundle movements can amplify a hair cell's response to oscillatory mechanical stimuli. Proc. Natl. Acad. Sci. U S A 96:14306-14311, 1999.

MiNOR LB, GOLDBERG JM. Vestibular-nerve inputs to the vestibuloocular reflex: A functional-ablation study in the squirrel monkey. J. Neurosci. 11(6):1636-1648, 1991.
NJeugna E, EichHorn JL, Kopp C, Harlicot P. Mechanics of the cupula: Effects of its thickness. J. Vestib. Res. 2:227-234, 1992.

Olson E. Observing middle and inner ear mechanics with novel intracochlear pressure sensors. J. Acoust. Soc. Am. 103(6):34453463, 1998.

OMAN CM, MARCUS EN, CURTHOYs IS. The influence of semicircular canal morphology on endolymph flow dynamics. Acta Otolaryngol. (Stockh.) 103:1-13, 1987.

PARKER DE. Labyrinth and cerebrospinal fluid pressure changes in guinea pigs and monkey during simulated zero $g$. Aviat. Space Environ. Med. 79:322-327, 1977.

PuRIa S, PeAKe WT, Rosowski JJ. Sound-pressure measurements in the cochlear vestibule of human-cadaver ears. J. Acoust. Soc. Am. 101(5):2754-2770, 1997.

RABBITT RD. Directional coding of three-dimensional movements by the vestibular semicircular canals. Biol. Cybern. 80:417-431, 1999.

RabBitT RD, BoYle R, Highstein SM. Sensory transduction of head velocity and acceleration in the toadfish horizontal semicircular canal. J. Neurophysiol. 72(2):1041-1048, 1994.

Rabbitt RD, Boyle R, Highstein SM. Mechanical indentation of the vestibular labyrinth and its relationship to head rotation in the toadfish, opsanus tau. J. Neurophysiol. 73(6):2237-2260, 1995.

RabitTt RD, Highstein SM, Boyle R. Determinants of semicircular canal afferent response dynamics in fish. Ann. N. Y. Acad. Sci. 781:213-243, 1996.

RABBitT RD, BoYLE R, HighSTEIN SM. Influence of surgical plugging on horizontal semicircular canal mechanics and afferent response dynamics. J. Neurophysiol. 82:1033-1052, 1999.

RabBitT RD, Boyle R, Highstein SM. Adaptation to maintained cupular displacements in semicircular canal hair cells vs. afferent nerves of the toadfish, Opsanus tau. Assoc. Res. Otolaryngol. Abstr. 582.

Ricci AJ, Crawford AC, FetTiPlace R. Active hair bundle motion linked to fast transducer adaptation in auditory hair cells. J. Neurosci. 20(19):7131-7142, 2000.

RÜSCH A, THURM U. Spontaneous and electrically induced movements of ampullary kinocilia and stereovilli. Hear. Res. 48:247264, 1990.

SALT AN. Fluid homeostasis in the inner ear. In: Harris JP, (ed) Ménière's disease. Kugler The Hague, Netherlands, 1999, p. 93-101.

SAlt AN, DeMott JE. Longitudinal endolymph flow associated with acute volume increase in the guinea pig cochlea. Hear. Res. 107:29-40, 1997.

SALt AN, DeMotT JE. Longitudinal endolymph movements induced by perilymphatic injections. Hear. Res. 123(1-2):137-147, 1998.

Silver RB, Reeves AP, Steinacker A, Highstein SM. Examination of the cupula and stereocilia of the horizontal semicircular canal in the toadfish Opsanus tau. Comp. Neurol. 402(1):48-61, 1998.

STEINHAUSEN W. Über die beobachtungen der cupula in den bognegängsampullen des labyrinthes des libenden hecths. Pflügers Arch. 232:500-512, 1933.

TONNDORF J. Vestibular signs and symptoms in Ménière's disorder: Mechanical considerations. Acta Otolaryngol. 95(5-6):421430, 1983

VANBUSKIRK WC. Vestibular mechanics. In: Skalak R, Chien S, (eds) Handbook of Bioengineering. McGraw-Hill, New York, 1987, p. 31.1-31.17

Wit HP, WARMerdam TJ, Albers WJ. Measurement of the mechanical compliance of the endolymphatic compartments in the guinea pig. Hear. Res. 145:82-90, 2000.

YAMAUCHI AM, RABbitT RD, Boyle R, Highstein SM. Displacement of the horizontal canal cupula in the toadfish. Assoc. Res. Otolaryngol. Abstr. 583. 